Article

\title{
Synthesis and Crystallographic Insight into the Structural Aspects of Some Novel Adamantane-Based Ester Derivatives
}

\author{
C. S. Chidan Kumar ${ }^{1,2, *}$, Huey Chong Kwong ${ }^{3}$, Siau Hui Mah ${ }^{4}$, Tze Shyang Chia ${ }^{\mathbf{1}}$, \\ Wan-Sin Loh ${ }^{1}$, Ching Kheng Quah ${ }^{1, *}$, Gin Keat Lim ${ }^{3}$, Siddegowda Chandraju 5 \\ and Hoong-Kun Fun ${ }^{1,6}$
}

1 X-ray Crystallography Unit, School of Physics, Universiti Sains Malaysia, Penang 11800, Malaysia; E-Mails: chiatzeshyang@hotmail.com (T.S.C.); wansin_loh@live.com (W.-S.L.); hkfun@usm.my (H.-K.F.)

2 Department of Chemistry, Alva's Institute of Engineering \& Technology, Visvesvaraya Technological University, Mijar, Moodbidri 574225, India

3 School of Chemical Sciences, Universiti Sains Malaysia, Penang 11800, Malaysia; E-Mails: stonekg01@gmail.com (H.C.K.); limgk@usm.my (G.K.L.)

4 School of Biosciences, Taylor's University, Lakeside Campus, Subang Jaya 47500, Selangor, Malaysia; E-Mail: siauhui.mah@taylors.edu.my

5 Department of Sugar Technology \& Chemistry, University of Mysore, Sir M.V. PG Center, Mandya 571402, Karnataka, India; E-Mail: chandraju1@yahoo.com

6 Department of Pharmaceutical Chemistry, College of Pharmacy, King Saud University, Riyadh 11451, Saudi Arabia

* Authors to whom correspondence should be addressed; E-Mails: chidankumar@gmail.com (C.S.C.K.); ckquah@usm.my (C.K.Q.); Tel.: +60-4-653-3888 (ext. 3690) (C.K.Q.);

Fax: +60-4-657-9150 (C.K.Q.).

Academic Editor: Derek J. McPhee

Received: 24 August 2015 / Accepted: 5 October 2015 / Published: 16 October 2015

\begin{abstract}
Adamantyl-based compounds are commercially important in the treatments for neurological conditions and type-2 diabetes, aside from their anti-viral abilities. Their values in drug design are chronicled as multi-dimensional. In the present study, a series of 2-(adamantan-1-yl)-2-oxoethyl benzoates, 2(a-q), and 2-(adamantan-1-yl)-2-oxoethyl 2-pyridinecarboxylate, $\mathbf{2 r}$, were synthesized by reacting 1 -adamantyl bromomethyl ketone with various carboxylic acids using potassium carbonate in dimethylformamide medium at room temperature. Three-dimensional structures studied using X-ray diffraction suggest
\end{abstract}


that the adamantyl moiety can serve as an efficient building block to synthesize 2-oxopropyl benzoate derivatives with synclinal conformation with a looser-packed crystal packing system. Compounds $2 \mathbf{a}, \mathbf{2 b}, \mathbf{2 f}, \mathbf{2 g}, \mathbf{2 i}, \mathbf{2 j}, \mathbf{2 m}, \mathbf{2 n}, \mathbf{2 0}, \mathbf{2 q}$ and $2 \mathbf{r}$ exhibit strong antioxidant activities in the hydrogen peroxide radical scavenging test. Furthermore, three compounds, $\mathbf{2 p}, \mathbf{2 q}$ and $\mathbf{2 r}$, show good anti-inflammatory activities in the evaluation of albumin denaturation.

Keywords: adamantyl; synclinal; crystal packing; antioxidant; anti-inflammatory

\section{Introduction}

Adamantane is the simplest diamondoid with a chemical formula of $\mathrm{C}_{10} \mathrm{H}_{16}$. It consists of four cyclohexane rings in an armchair configuration. To date, seven adamantane derivatives have been applied in clinical usage to treat acne vulgaris [1], Alzheimer's disease [2], as an anti-viral [3-7] and for type-2 diabetes [8-10], while the others are in development as potential therapeutics for iron overload disease, neurological conditions, malaria, tuberculosis and cancers [11]. Previous studies on adamantyl-based compounds revealed their antioxidant and anti-inflammatory properties [12-14]. As a reactive oxygen species, hydrogen peroxide is generated as a by-product of biological reaction. These reactive species will cause oxidative damaging effects in living cells. Hydrogen peroxide acts as a weak oxidizing agent and reacts with $\mathrm{Fe}^{2+}$ or $\mathrm{Cu}^{2+}$ ions to form the hydroxyl radical [15]. Similarly, free radicals, such as DPPH (2,2-diphenyl-1-picrylhydrazyl), are readily attacked and promote oxidative damage of biomolecules, such as lipids, proteins and DNA, leading to many human pathological conditions [16,17]. On the other hand, failure in the downregulation of pro-inflammatory mediators will result in an imbalance between inflammation and its regulation, which leads to chronic inflammation due to excessive macrophage responses. The resulting disease conditions from chronic inflammation include asthma, rheumatic arthritis, atherosclerosis and cancer [18]. Protection against protein denaturation, a well-documented phenomenon caused by the inflammation process, is the main consideration in developing non-steroidal anti-inflammatory drugs (NSAIDs) [19].

Encouraged by the biological activities of adamantane derivatives, we herein present FTIR, ${ }^{1} \mathrm{H}-\mathrm{NMR}$ and ${ }^{13} \mathrm{C}$-NMR spectra and single-crystal X-ray diffraction analysis of some new adamantyl-based ester derivatives. Using a group comparison of single-crystal X-ray diffraction data, the structural conformation, structural occupancy and crystal packing similarity were studied. Their antioxidant activities were evaluated by hydrogen peroxide and the DPPH radical scavenging assay. In addition, the anti-inflammatory activity of these adamantyl-based ester derivatives was determined by the protein denaturation assay.

\section{Results and Discussion}

The full reaction involved the synthesis of 1-adamantyl bromomethyl ketone, $\mathbf{1}$, which is essential for subsequent synthesis of $\mathbf{2}(\mathbf{a}-\mathbf{r})$ in accordance with the reported procedure [20], as shown in Scheme 1. The structures of the intermediate and final compounds were elucidated by various spectral techniques, like FTIR, ${ }^{1} \mathrm{H}-\mathrm{NMR},{ }^{13} \mathrm{C}-\mathrm{NMR}$ and single-crystal X-ray diffraction analysis. The asymmetric and 
symmetric stretchings of the methyl group (asym $2960 \mathrm{~cm}^{-1}$ : sym $2870 \mathrm{~cm}^{-1}$ ) in $\mathbf{1}$ had changed to methylene stretchings (asym $2920 \mathrm{~cm}^{-1}$ : sym $2850 \mathrm{~cm}^{-1}$ ); however, the synthesis of 1 is confirmed by ${ }^{1} \mathrm{H}-\mathrm{NMR}$ when the peak corresponding to the methyl group of 1-adamantyl methyl ketone is replaced by the peak corresponding to the methylene group near $\delta 4.1 \mathrm{ppm}$. Esterification of $\mathbf{2}(\mathbf{a}-\mathbf{r})$ was confirmed by FTIR, where the peak corresponding to the hydroxyl of carboxylic acid disappeared. Furthermore, some peaks were observed at $\sim 1300 \mathrm{~cm}^{-1}-1020 \mathrm{~cm}^{-1}$, which corresponded to $\mathrm{C}-\mathrm{O}$ stretching of ester. Similarly, the ${ }^{1} \mathrm{H}-\mathrm{NMR}$ of $\mathbf{2}(\mathbf{a}-\mathbf{r})$ displays a singlet corresponding to the methylene group at higher chemical shifts $(\delta 5.1 \mathrm{ppm})$ associated with the other required aromatic peaks. In the FTIR spectra of $\mathbf{2}(\mathbf{a}-\mathbf{r})$, the exceptional and predictable observations are the occurrences of $\mathrm{N}=\mathrm{O}$ stretching in 2(I- $\mathbf{m})$ and the $\mathrm{N}-\mathrm{H}$ stretching in $\mathbf{2}(\mathbf{o}-\mathbf{q})$ at $\sim 1530 \mathrm{~cm}^{-1}$ and $\sim 3490 \mathrm{~cm}^{-1}$, respectively; whereas for ${ }^{13} \mathrm{C}-\mathrm{NMR}$, the constant appearances of carbonyl carbons peaks at 207 and 165 ppm further support the formation of the ester bond in Compounds $\mathbf{2}(\mathbf{a}-\mathbf{r})$. The crystal structure for all compounds, except $\mathbf{2 m}$ and $\mathbf{2 q}$, were determined by single-crystal X-ray diffraction analysis. All spectra, crystallography data and crystal packing are described in detail in the Supplementary Data.

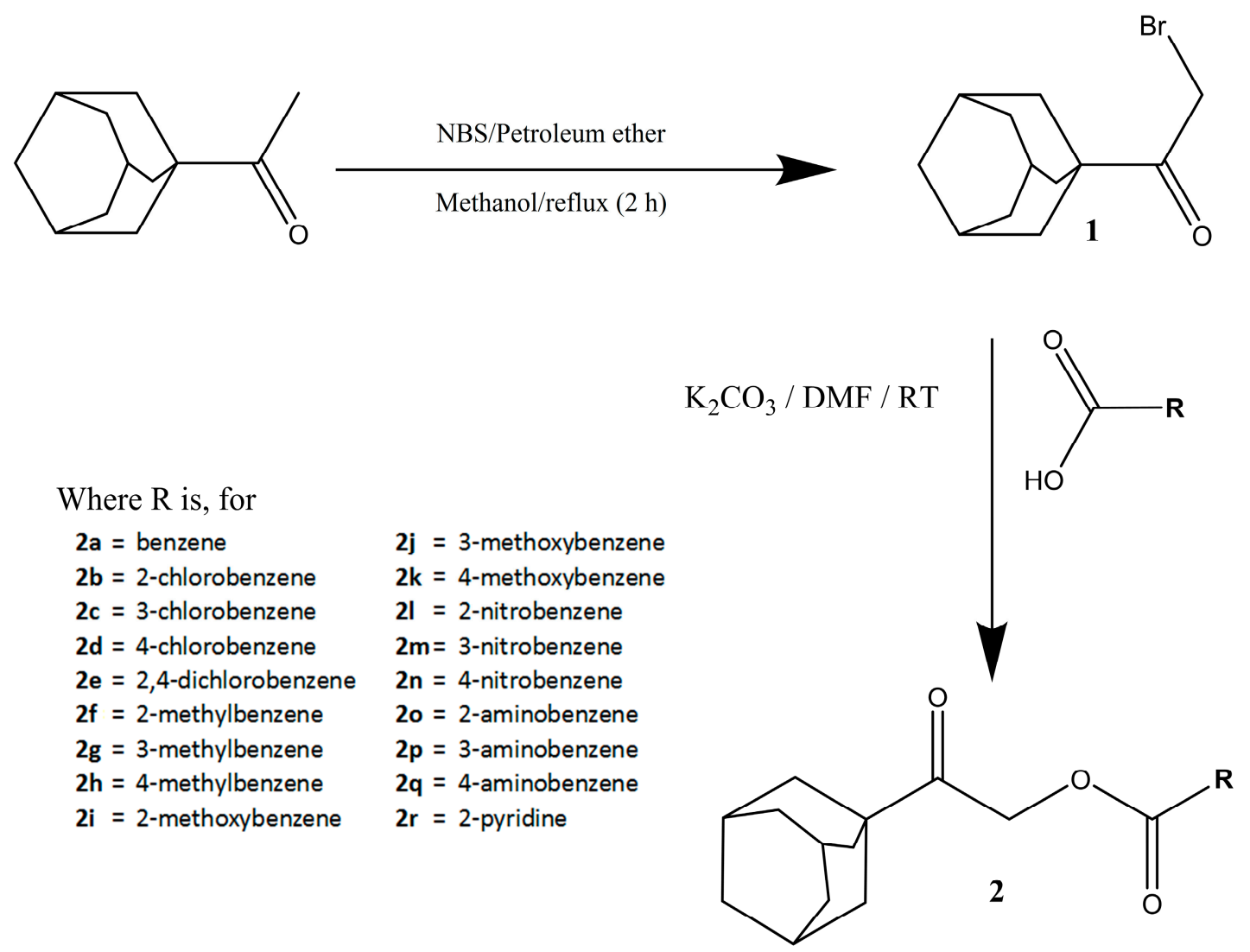

Scheme 1. Reaction scheme for $\mathbf{2}(\mathbf{a}-\mathbf{r})$.

\subsection{General Description of the Crystal Structure Conformations}

The asymmetric unit $\left(Z^{\prime}\right)$ of the studied compounds consists of either one $(\mathbf{2} \mathbf{c}, \mathbf{2} \mathbf{d}, \mathbf{2} \mathbf{g}, \mathbf{2} \mathbf{h}, \mathbf{2} \mathbf{j}, \mathbf{2} \mathbf{k}$, $\mathbf{2 n}-\mathbf{p}$ and $\mathbf{2 r})$ or two (2a, 2b, 2e, 2f, $\mathbf{2} \mathbf{i}$ and $\mathbf{2 l})$ crystallographic independent molecules. For a crystal with $Z^{\prime}=2$, the independent molecules are hereafter denoted as Molecules $A$ and $B$, respectively. The orientational disorder of the adamantane group is observed in $\mathbf{2 a}, \mathbf{2 b}, \mathbf{2} \mathbf{f}, \mathbf{2 j}, \mathbf{2} \mathbf{l}$ and $\mathbf{2 0}$ with a refined 
site occupancy ratio, as summarized in the table below (Table 1). All disordered components of adamantane are rotationally-related, and in particular, two-fold rotation disorder $\left(c a .180^{\circ}\right)$ is observed in 2a (Molecule $B$ ) and $\mathbf{2 f}$ (Molecule $B$ ).

Table 1. Refined site occupancy ratio for disordered compounds.

\begin{tabular}{cl}
\hline Compound & \multicolumn{1}{c}{ Refined Site Occupancy Ratio } \\
\hline \multirow{2}{*}{$\mathbf{a}$} & $0.390(10): 0.610(10)(A)$ \\
& $0.695(4): 0.305(4)(B)$ \\
\hline $\mathbf{2 b}$ & $0.431(10): 0.569(10)(A)$ \\
\hline \multirow{2}{*}{$\mathbf{2 f}$} & $0.716(6): 0.284(6)(A)$ \\
& $0.793(4): 0.207(4)(B)$ \\
\hline $\mathbf{2 j}$ & $0.753(3): 0.247(3)$ \\
$\mathbf{2 l}$ & $0.873(4): 0.127(4)(B)$ \\
$\mathbf{2 o}$ & $0.897(4): 0.103(4)$ \\
\hline
\end{tabular}

Molecular conformation of these compounds can be characterized by three degrees of freedom, which are the torsion angles of $\mathrm{C} 1-\mathrm{C} 8-\mathrm{C} 11-\mathrm{O} 2(\tau 1), \mathrm{C} 11-\mathrm{C} 12-\mathrm{O} 1-\mathrm{C} 13(\tau 2)$ and $\mathrm{O} 1-\mathrm{C} 13-\mathrm{C} 14-\mathrm{C} 15$ ( $\tau 3)$ (Figure 1). Although the torsion angle between the adamantane moiety and the adjacent carbonyl group $(\mathrm{C} 1-\mathrm{C} 8-\mathrm{C} 11-\mathrm{O} 2, \tau 1)$ is one of the degrees of freedom, it was irrelevant for the comparison due to its randomness and the effect of disordered orientations. By referring to the previous report [20], torsion angle $\tau 2$, which interconnects two carbonyl groups, tends to adopt two types of conformations, either synclinal or periplanar. However, all structures reported in this study adopt only one conformation in which $\tau 2$ torsion angles are all in synclinal conformation ranging from $70^{\circ}-86^{\circ}$, which may be due to the introduction of the bulky adamantane moiety. The range of the $\mathrm{O} 1-\mathrm{C} 13-\mathrm{C} 14-\mathrm{C} 15 \tau 3$ torsion angle, which defines the twisted angle between the carboxylate group and the six-membered ring, is relatively larger compared to $\tau 2$, ranging from $1.25^{\circ}-39.08^{\circ}$ and $126.4^{\circ}-179.57^{\circ}$. In compounds $2 \mathrm{e}$ (2,4-dichlorobenzene), $\mathbf{2} \mathbf{i}$ (2-methoxybenzene) and $\mathbf{2} \mathbf{l}$ (2-nitrobenzene), the $\tau 3$ is probably induced by the steric repulsion between the $o$-substituent and the adjacent carbonyl oxygen atom. Nevertheless, exceptional cases are observed in $\mathbf{2 b}$ (2-chlorobenzene), $\mathbf{2} \mathbf{f}$ (2-methylbenzene) and $\mathbf{2 0}$ (2-aminobenzene) with small deviations of $3.78^{\circ}, 11.55^{\circ}, 6.64^{\circ}, 9.58^{\circ}$ and $8.08^{\circ}$ from perfect planarity. The amino substituent at the -ortho position (2o) forms a strong intramolecular $\mathrm{N}-\mathrm{H} \cdots \mathrm{O}$ hydrogen bond with the adjacent carbonyl group to improve the planarity of the benzoate group $\left(\tau 3=171.92^{\circ}\right)$. Torsion angles between two carbonyl group $(\mathrm{C} 11-\mathrm{C} 12-\mathrm{O} 1-\mathrm{C} 13, \tau 2)$ and torsion angles between the carbonyl group with its adjacent benzene ring $(\mathrm{O} 1-\mathrm{C} 13-\mathrm{C} 14-\mathrm{C} 15, \tau 3)$ of the reported compounds are summarized in Table 2.

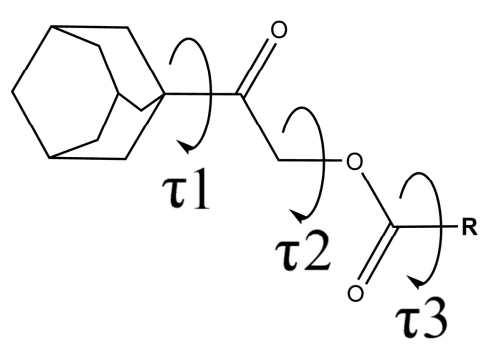

Figure 1. General chemical scheme for all compounds, showing $\tau 1, \tau 2$ and $\tau 3$ as torsion angles. 
Table 2. Summary of the $\mathrm{C} 11-\mathrm{C} 12-\mathrm{O} 1-\mathrm{C} 13$ and $\mathrm{O} 1-\mathrm{C} 13-\mathrm{C} 14-\mathrm{C} 15$ torsion angles.

\begin{tabular}{cccc}
\hline \multirow{2}{*}{ Compound } & Substituent & $\begin{array}{c}\text { Torsion Angles } \\
\mathbf{C 1 1 - O 1 - C 1 3 , \tau 2}\end{array}$ & $\begin{array}{c}\text { Torsion Angles } \\
\text { O1-C13-C14-C15, } \mathbf{\tau 3}\end{array}$ \\
\hline $\mathbf{2 a}$ & Benzene & $-81.31,75.84$ & $-9.95,2.84$ \\
$\mathbf{2 b}$ & 2-Chlorobenzene & $78.96,75.23$ & $11.55,-3.78$ \\
$\mathbf{2 c}$ & 3-Chlorobenzene & 73.10 & 3.22 \\
$\mathbf{2 d}$ & 4-Chlorobenzene & 73.75 & -179.57 \\
$\mathbf{2 e}$ & 2,4-Dichlorobenzene & $-69.7,-85.50$ & $138.13,135.26$ \\
$\mathbf{2 f}$ & 2-Methylbenzene & $-76.09,77.77$ & $-9.58,6.64$ \\
$\mathbf{2 g}$ & 3-Methylbenzene & -72.91 & 1.25 \\
$\mathbf{2 h}$ & 4-Methylbenzene & 75.42 & -170.83 \\
$\mathbf{2 i}$ & 2-Methoxybenzene & $-86.12,-79.34$ & $39.08,156.87$ \\
$\mathbf{2 j}$ & 3-Methoxybenzene & -75.05 & -173.58 \\
$\mathbf{2 k}$ & 4-Methoxybenzene & -76.60 & 168.36 \\
$\mathbf{2 1}$ & 2-Nitrobenzene & $-70.96,-70.23$ & $126.4,130.00$ \\
$\mathbf{2 n}$ & 4-Nitrobenzene & 73.57 & -1.86 \\
$\mathbf{2 0}$ & 2-Aminobenzene & -76.5 & 171.92 \\
$\mathbf{2} \mathbf{p}$ & 3-Aminobenzene & 77.13 & -17.86 \\
$\mathbf{2 r}$ & 2-Pyridine & -75.07 & $-17.79,22.32$ \\
\hline
\end{tabular}

\subsection{Structural Occupancy and Crystal Packing Similarity}

Sixteen present adamantyl-based ester derivatives and thirty-six reported phenacyl benzoate derivatives, which are found from the Cambridge Structure Database (CSD 5.35) search, are compared to each other in order to identify the effect of the replacement of the electron-rich phenyl ring (search result) with the bulky adamantane moiety (present compounds) or vice versa on the molecular conformation and structural occupancy. In the aspect of molecular conformation, it is noteworthy to observe that neither periplanar conformation nor mixed (periplanar and synclinal) conformation is adopted by the currently studied compounds, unlike the variations displayed in phenacyl benzoates. All present compounds adopt synclinal conformation with $\mathrm{C} 13-\mathrm{O} 1-\mathrm{C} 12-\mathrm{C} 11$ torsion angles falling in the range from $69.7^{\circ}-86.12^{\circ}$, which is comparable to those previously reported for phenacyl benzoate derivatives that adopt the same conformation $\left(71^{\circ}-91^{\circ}\right)[20]$. The structural occupancies of all present and reported compounds are listed in Table 3. The structural occupancy of phenacyl benzoate derivatives has a larger range compared to adamantyl-based ester derivatives and is mostly in between $63 \%$ and $69 \%$ (30 out of 36). All of the present adamantyl-based compounds are below this range, except $2 \mathbf{n}$ (Figure 2). The existence of the adamantane moiety in the present compounds not only reduces the weak intermolecular $\pi \ldots \pi$ or $\mathrm{C}-\mathrm{H} . . . \pi$ interactions as compared to phenacyl benzoates, it also limits the packing patterns of adamantyl-based ester derivatives as indicated by the high occurrence of isostructures. 
Table 3. List of the structural occupancy of the present and reported compounds ${ }^{\mathrm{i}}$.

\begin{tabular}{cccccc}
\hline Compound & $\begin{array}{c}\text { Packing } \\
\text { Coefficient (\%) }\end{array}$ & Compound & $\begin{array}{c}\text { Packing } \\
\text { Coefficient (\%) }\end{array}$ & Compound & $\begin{array}{c}\text { Packing } \\
\text { Coefficient (\%) }\end{array}$ \\
\hline $\mathbf{2 a}$ & 61.11 & CIXVUC [20] & 63.94 & GITHUN [21] & 64.40 \\
$\mathbf{2 b}$ & 61.32 & CIXWAJ [20] & 64.33 & MANGIR [22] & 61.06 \\
$\mathbf{2 c}$ & 62.33 & CIXWEN [20] & 62.08 & OBOYIP [23] & 67.22 \\
$\mathbf{2 d}$ & 62.53 & CIXWIR [20] & 63.98 & OCAKUA [24] & 63.92 \\
$\mathbf{2 e}$ & 60.79 & CIYCAQ [20] & 67.27 & OCAQUG [25] & 66.98 \\
$\mathbf{2 f}$ & 60.97 & CIYCEU [20] & 65.07 & OCEFEJ [26] & 68.55 \\
$\mathbf{2 g}$ & 61.68 & CIYCIY [20] & 68.83 & PECZAA [27] & 64.37 \\
$\mathbf{2 h}$ & 61.02 & CIYCOE [20] & 62.96 & PODQIK [28] & 60.66 \\
$\mathbf{2 i}$ & 61.58 & CIYFUN [20] & 62.38 & PODRAD [29] & 63.77 \\
$\mathbf{2 j}$ & 61.08 & CIYGAU [20] & 64.89 & USIWID [30] & 62.53 \\
$\mathbf{2 k}$ & 61.07 & EVAFOX [31] & 68.00 & USIWOJ [32] & 66.04 \\
$\mathbf{2 1}$ & 61.85 & EVAJAN [33] & 65.64 & VOBYUI [34] & 63.97 \\
$\mathbf{2 n}$ & 63.05 & EVAJIV [35] & 64.03 & YAFWEJ [36] & 66.25 \\
$\mathbf{2 0}$ & 61.24 & EVAZEH [37] & 63.03 & YAFZAI [38] & 68.65 \\
$\mathbf{2 p}$ & 61.81 & EVEGIW [39] & 63.25 & YAHGUL [40] & 63.55 \\
$\mathbf{2 r}$ & 60.61 & EVEGOC [41] & 63.22 & YAHYOX [42] & 63.37 \\
AZULUD [43] & 63.85 & EVEVEH [44] & 63.04 & & \\
CIQNEW [45] & 64.07 & GARCEJ [46] & 65.80 & & \\
\hline
\end{tabular}

${ }^{\mathrm{i}}$ Each reported compound is represented by the Cambridge Crystallographic Data Centre (CCDC) reference code, and its systematic name is provided in the Supplementary Materials.

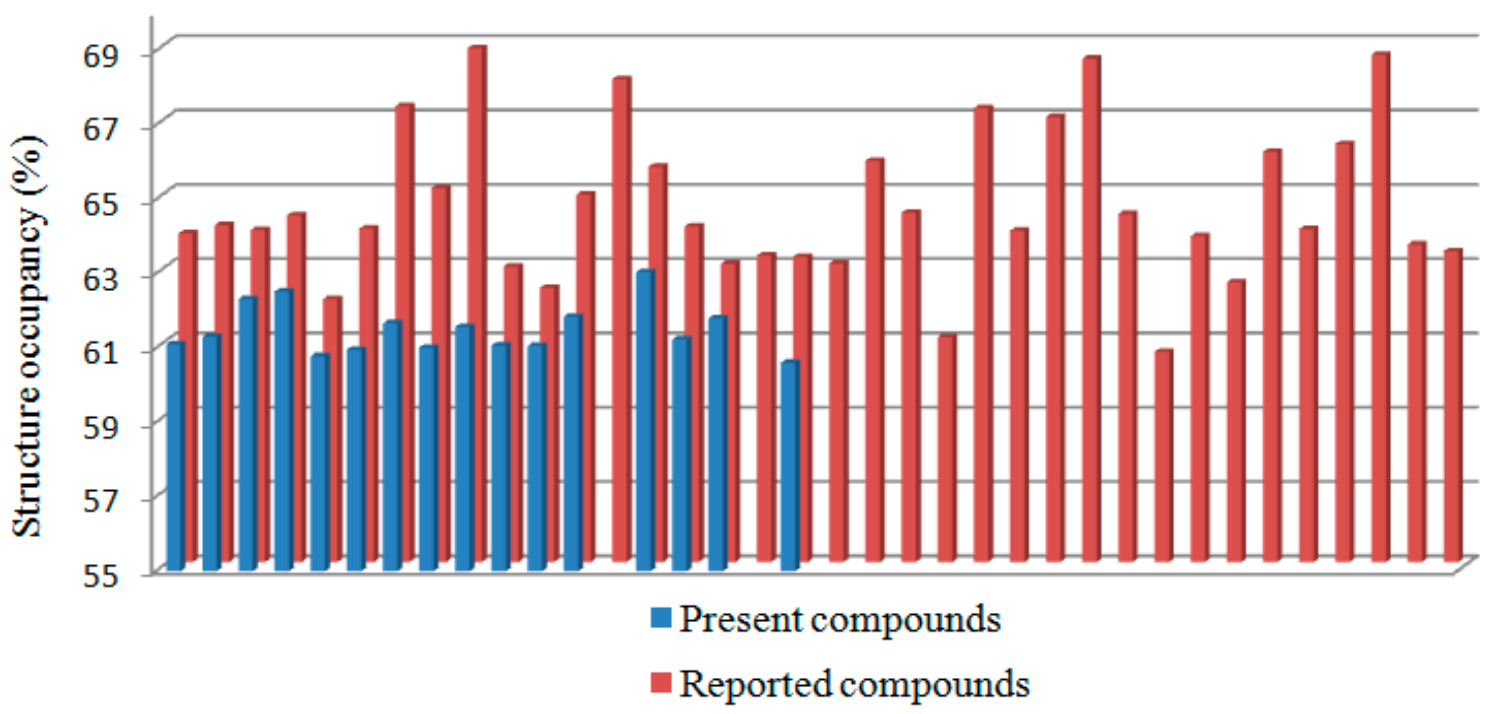

Figure 2. Structural occupancy comparison of the present and reported compounds.

In the investigation of the crystal structural similarity among the current compounds, there are four pairs of compounds ( $2 \mathbf{a} / \mathbf{2 f}, \mathbf{2} \mathbf{c} / \mathbf{2 g}, \mathbf{2 h} / \mathbf{2} \mathbf{k}$ and $\mathbf{2 d} / \mathbf{2 n})$ found, and each pair is crystallized in the same space group with similar lattice constants, which are two main characteristics of 3D structural similarity. Indeed, these four pairs of compounds show isostructural relationships with a similar packing pattern, as shown in their overlaid crystal structure diagrams (Figures 3-6, respectively). Besides that, 2D similarities are 
observed in $\mathbf{2 b} / \mathbf{2 a}$ and $\mathbf{2 b} / \mathbf{2 f}$, while Compounds $\mathbf{2 c}, \mathbf{2 d}, \mathbf{2 g}$ and $\mathbf{2 n}$ show 2D similarity among each other (Figure 7).

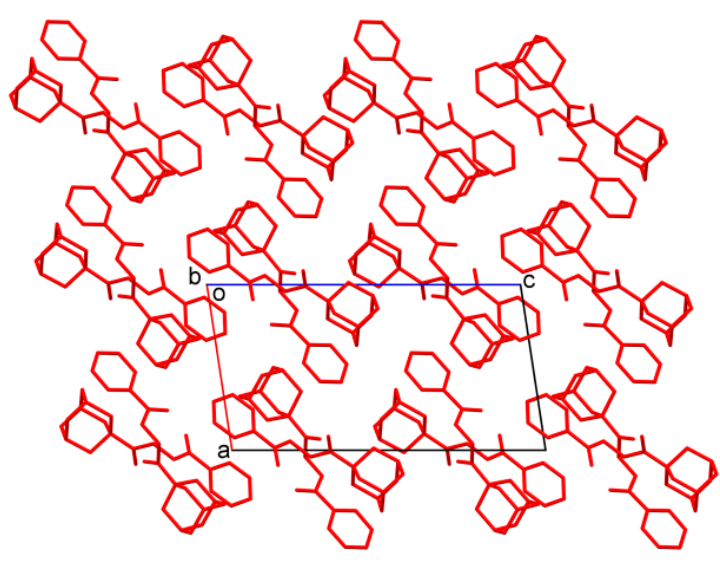

$2 a$

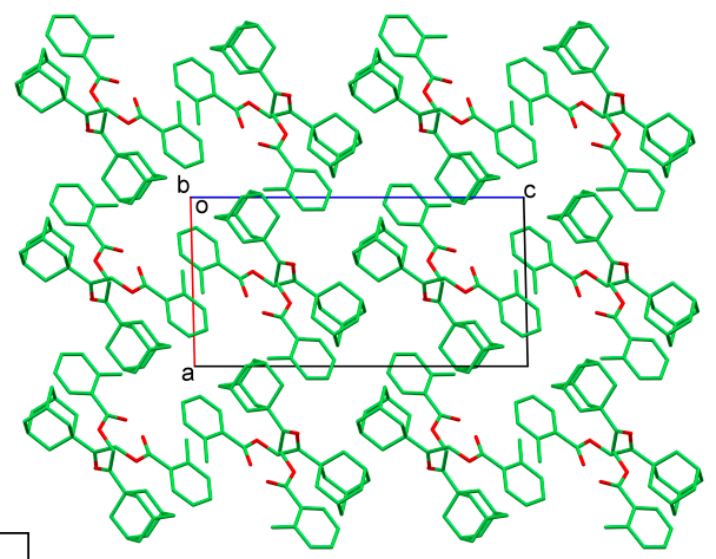

$2 f$

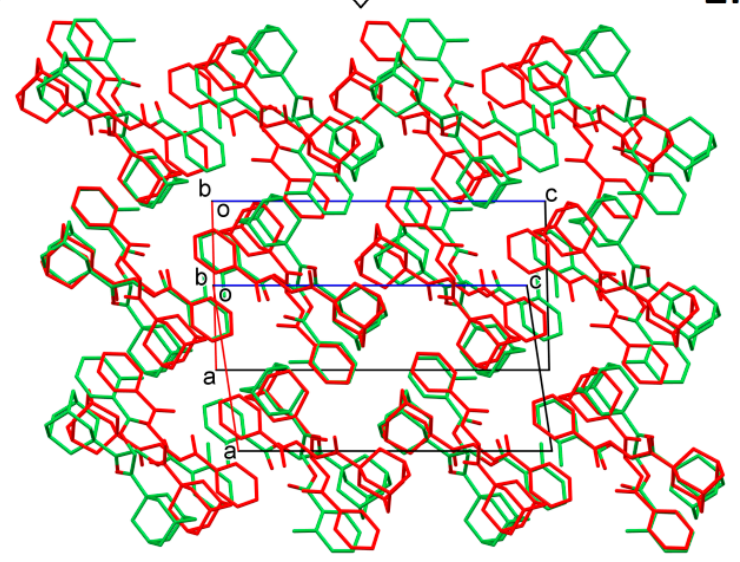

Figure 3. Crystal packing comparison of Compounds 2a and $\mathbf{2 f}$.
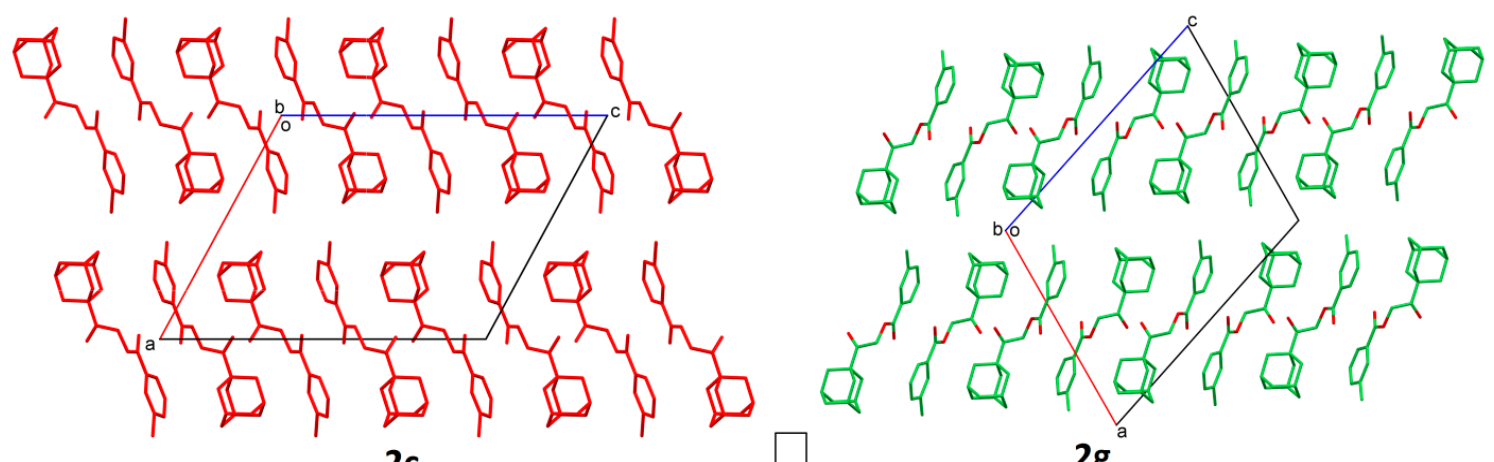

2c

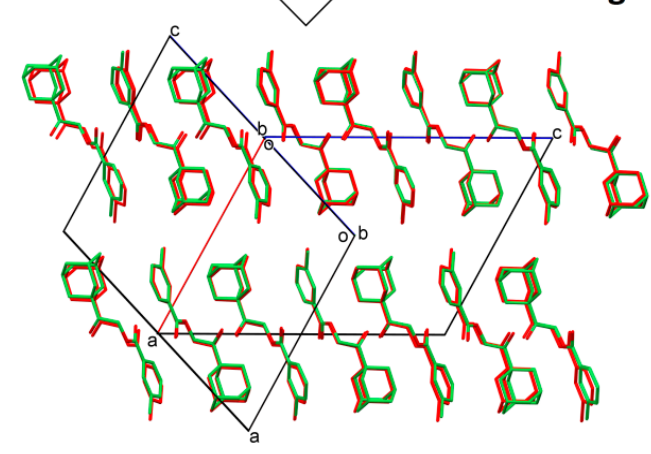

Figure 4. Crystal packing comparison of Compounds $\mathbf{2 c}$ and $\mathbf{2 g}$. 


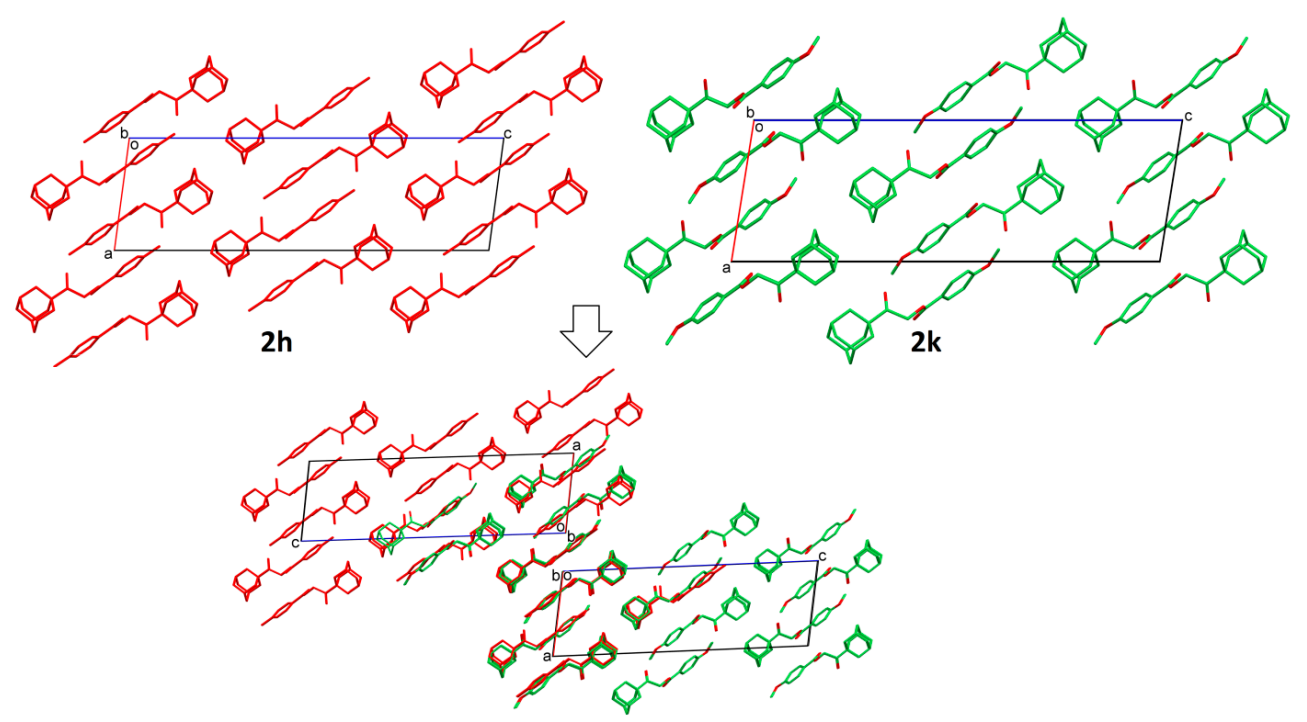

Figure 5. Crystal packing comparison of Compounds $\mathbf{2 h}$ and $\mathbf{2 k}$.

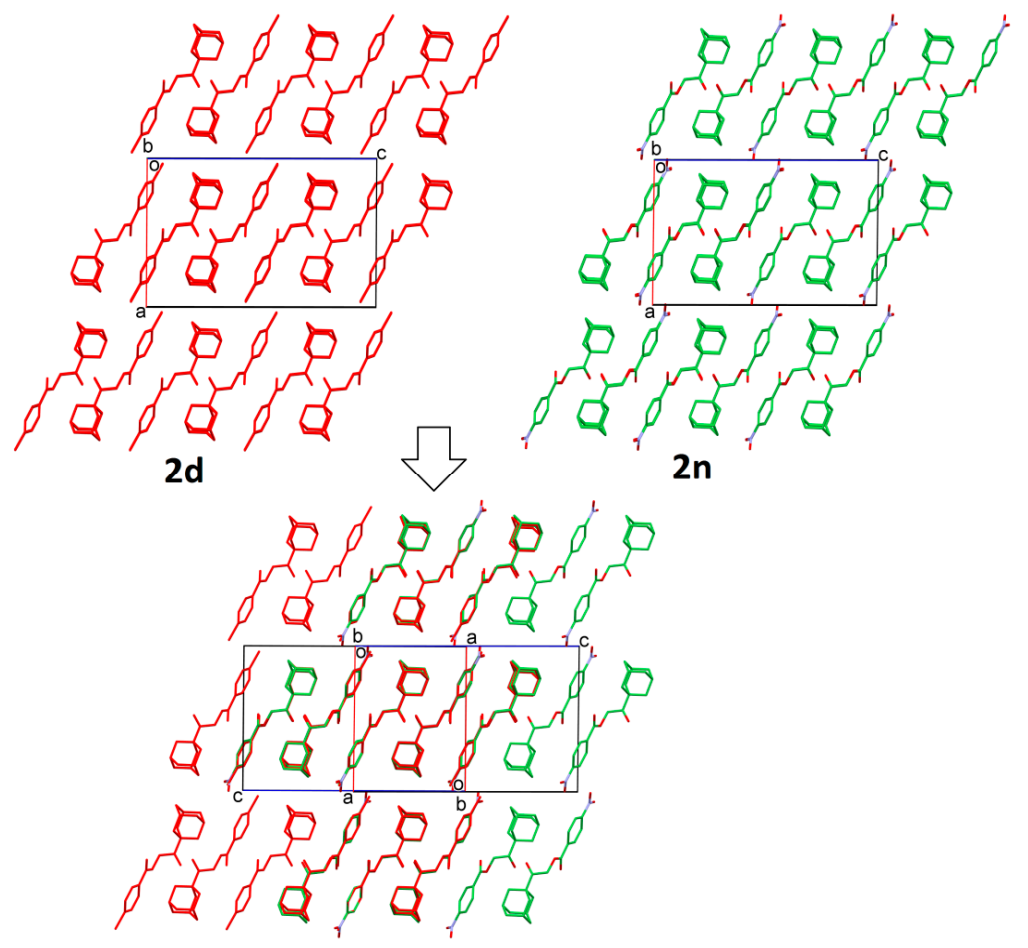

Figure 6. Crystal packing comparison of Compounds $\mathbf{2 d}$ and $\mathbf{2 n}$.
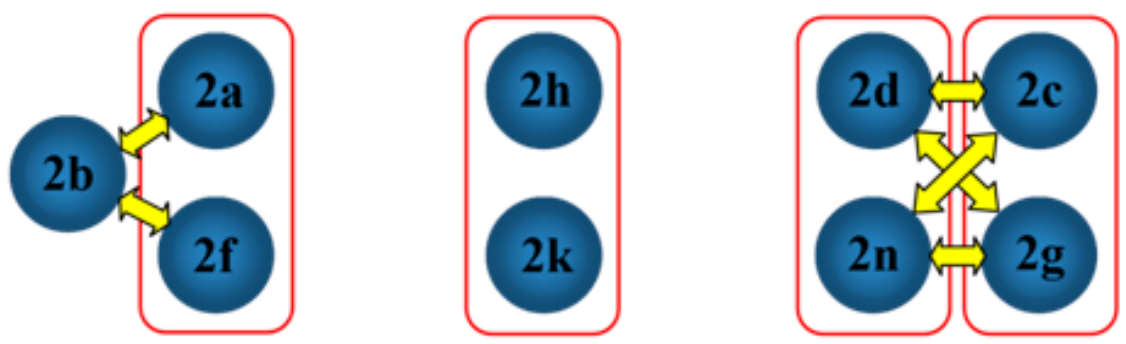

Figure 7. Crystal packing relationship in some studied compounds. Red boxes and yellow arrows indicate $3 \mathrm{D}$ and $2 \mathrm{D}$ similarities, respectively. 


\subsection{Antioxidant and Anti-Inflammatory Properties}

The antioxidant properties of adamantane-based compounds were determined by two methods, which are hydrogen peroxide and DPPH radicals scavenging abilities. The scavenging effects of hydrogen peroxide by the adamantyl-based compounds were evaluated at a concentration of $250 \mu \mathrm{g} / \mathrm{mL}$ and are summarized in Figure 8. Eleven adamantane derivatives show positive scavenging effects on $\mathrm{H}_{2} \mathrm{O}_{2}$. Compound $\mathbf{2 b}$ possesses the strongest scavenging activity, which is $48.55 \%$, followed by Compounds $\mathbf{2 q}$ and $2 \mathrm{~g}$ with $42.96 \%$ and $42.56 \%$, respectively. The scavenging activities of these compounds are comparable to the standard compound, ascorbic acid, with an inhibition percentage of $43.38 \%$. On the other hand, adamantyl-based compounds did not show positive DPPH radical scavenging properties at a concentration of $1000 \mu \mathrm{g} / \mathrm{mL}$. The results show that adamantane derivatives are selective to the inhibition of hydrogen peroxide radicals.

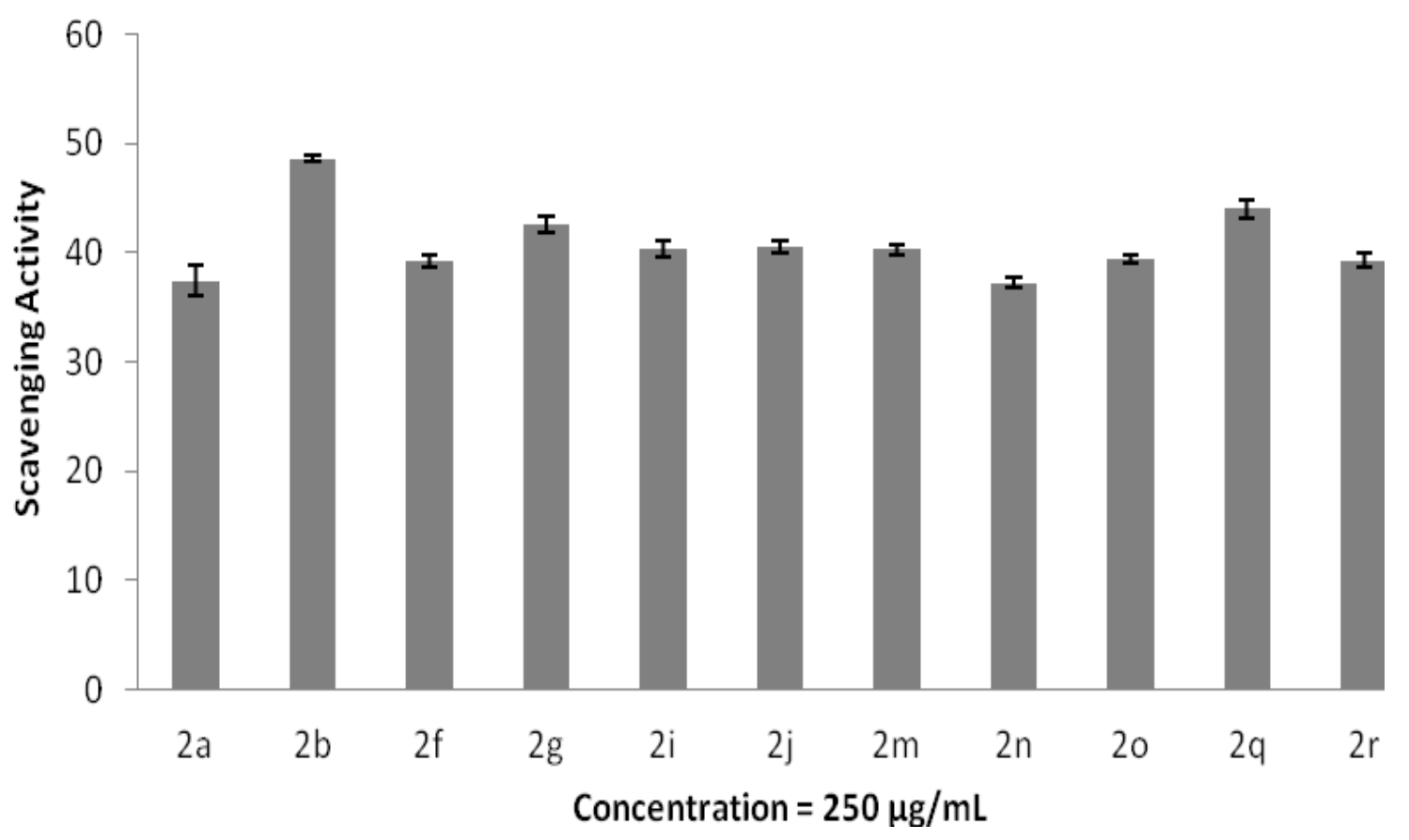

Figure 8. Hydrogen peroxide radical scavenging. The data represent the percentage of hydrogen peroxide radical inhibition (mean $\pm \mathrm{SD}$ ), and experiments were performed in triplicate.

The anti-inflammatory effects of adamantyl-based compounds at a concentration of $250 \mu \mathrm{g} / \mathrm{mL}$ were evaluated by the protein denaturation assay. The results of the percentage of inhibition against protein denaturation are presented in Figure 9. Compounds $\mathbf{2 p}, \mathbf{2 q}$ and $\mathbf{2 r}$ are strong protein denaturation inhibitors with inhibition percentages of $41.92 \%, 43.94 \%$ and $40.91 \%$, respectively, which are better than the standard drug, diclofenac sodium, with an inhibition percentage of $37.88 \%$. These three compounds consist of either an amino-substituted phenyl ring or a pyridine ring. Hence, the high inhibition effects are deduced to be the direct contribution from the nitrogen-containing group in adamantyl-based compounds. 


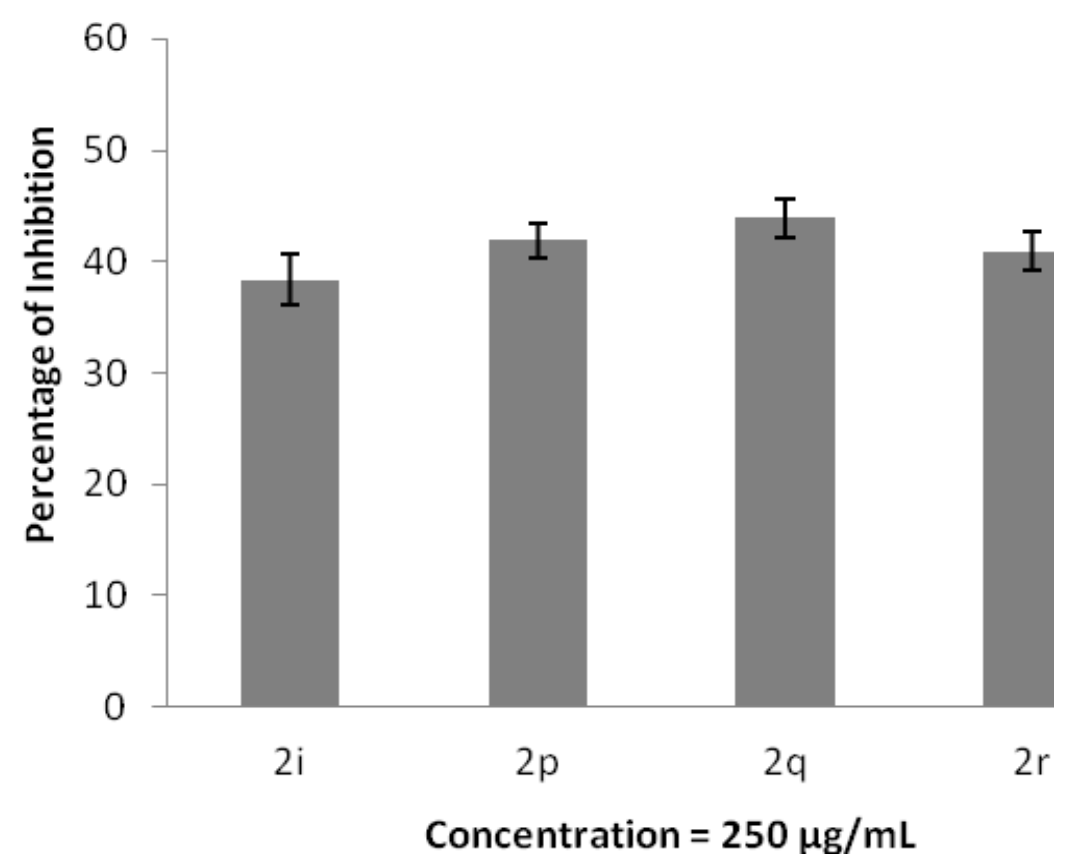

Figure 9. Inhibition of protein denaturation. The data represent the percentage of hydrogen peroxide radical inhibition (mean $\pm \mathrm{SD}$ ), and experiments were performed in triplicate.

\section{Experimental Section}

The reagents and solvents for the synthesis work were obtained commercially from Sigma Aldrich Corporation (St. Louis, MO, USA) and used without any additional purification. Melting points were determined on a Stuart (Staffordshire, UK) SMP10 apparatus. ${ }^{1} \mathrm{H}$ and ${ }^{13} \mathrm{C}$ nuclear magnetic resonance (NMR) spectra were determined in $\mathrm{CDCl}_{3}$ at $500 \mathrm{MHz}$ and $125 \mathrm{MHz}$, respectively, using a Bruker Advance III 500 spectrometer (Bruker Corporation, Billerica, MA, USA). Fourier transform infrared spectroscopy (FTIR) spectra were recorded on a Perkin Elmer Frontier FTIR spectrometer (PerkinElmer, Inc., Waltham, MA, USA) equipped with attenuated total reflection (ATR).

The X-ray analysis for all samples was performed using a Bruker APEX II DUO CCD diffractometer (Bruker Corporation) employing MoK $\alpha$ radiation $(\lambda=0.71073 \AA$ ) with $\varphi$ and $\omega$ scans. X-ray data for all compounds were collected at room temperature. Data reduction and absorption correction were performed using the SAINT and SADABS program [47]. All structures were solved by the direct method and refined by full-matrix least-squares techniques on $F^{2}$ using the SHELXTL software package [48]. All non-hydrogen atoms were refined anisotropically, except for the minor disordered components of $\mathbf{2} \mathbf{I}$ and $\mathbf{2 0}$ with a site occupancy of less than 0.2. The C-bound H atoms were calculated geometrically with isotropic displacement parameters set to 1.2-times the equivalent isotropic $U$ value of the parent carbon atoms. N-bound $\mathrm{H}$ atoms are located by the difference Fourier map and refined freely $(\mathrm{N}-\mathrm{H}=0.77$ (5) -0.87 (6) $\AA$ ). A similar geometry restraint (SAME) was applied to the disordered adamantane moiety in $\mathbf{2 f}$ and $\mathbf{2 o}$ and also the full molecule in 2r. Packing coefficient (\%)/structure occupancy was calculated using the Olex2 program with the command (-calcvoid) [49]. Crystallographic data for the structures reported in this paper (2a-r, excluding $\mathbf{2} \mathbf{m}$ and $\mathbf{2 q})$ have been deposited with the Cambridge Crystallographic Data Centre (CCDC) as supplementary publication. CCDC 1030854-1030869 contain the supplementary crystallographic data for this paper. These data can be obtained free of charge 
via http://www.ccdc.cam.ac.uk/conts/retrieving.html (or from the CCDC, 12 Union Road, Cambridge CB2 1EZ, UK; Fax: +44 1223 336033; E-mail: deposit@ccdc.cam.ac.uk).

\subsection{Synthesis}

1-Adamantyl methyl ketone was refluxed with $N$-bromo succinimide and petroleum ether in methanol at $333 \mathrm{~K}$ for two hours. The resultant 1-adamantyl bromomethyl ketone (1) precipitate was filtered and recrystallized with ethanol. After that, 1-adamantyl bromomethyl ketone (1) (0.51 g, 0.002 mol) was reacted with the corresponding carboxylic acid $(0.003 \mathrm{~mol})$ with the presence of potassium carbonate in DMF $(8 \mathrm{~mL})$ and stirred at room temperature for about $3 \mathrm{~h}$. The reaction progress was monitored by thin layer chromatography (TLC). After the reaction completed, the reaction mixture was poured into ice-cooled water and kept stirring for $10 \mathrm{~min}$. The solid obtained was filtered out, washed successively with distilled water and recrystallized from acetone after it dried [20]. All targeted compounds were synthesized in good yield and high purity. Suitable single-crystal specimens were obtained from various types of solvents, as described below. The chemical structures were characterized by using FTIR and NMR spectroscopies. The crystal structures for all compounds except $\mathbf{2} \mathbf{m}$ and $\mathbf{2 q}$ were determined by single-crystal X-ray diffraction analysis.

\subsection{Spectroscopic Details}

1-Adamantyl bromomethyl ketone (1): Solvent for growing crystal: ethanol; yield: 85\%; M.P. 326-328 K; FTIR (ATR (solid) cm $\mathrm{cm}^{-1}$ ): 2905, 2851 (C-H, v), 1709 (C=O, v), 1254, 1094, 1063 (C-O, v), 733 (C-Cl, v); ${ }^{1} \mathrm{H}-\mathrm{NMR}\left(500 \mathrm{MHz}, \mathrm{CDCl}_{3}\right.$ ): $\delta$ ppm 4.18 (s, 2H, - $\mathrm{CH}_{2}$ ), 2.09 (br-s, 3H, adamantane-CH), 1.91-1.90 (br-d, 6H, adamantane- $\mathrm{CH}_{2}$ ), $1.80-1.72$ (br-q, 6H, adamantane- $\left.\mathrm{CH}_{2}\right) ;{ }^{13} \mathrm{C}-\mathrm{NMR}\left(125 \mathrm{MHz}, \mathrm{CDCl}_{3}\right)$ : $\delta$ ppm $205.57(\mathrm{C}=\mathrm{O}), 46.62\left(-\mathrm{CH}_{2}\right), 38.53,36.34,31.83,27.83$ (adamantane-Cs).

2-(Adamantan-1-yl)-2-oxoethyl benzoate (2a): Solvent for growing crystal: acetone; yield: 80\%; M.P. 374-376 K; FTIR (ATR (solid) cm $\mathrm{cm}^{-1}$ ): 2917, 2850 (C-H, v), 1709 (C=O, v), 1602, 1413 (Ar, C=C, v), 1277, 1120 (C-O, v), 706 (C-H, $\omega)$; ${ }^{1} \mathrm{H}-\mathrm{NMR}\left(500 \mathrm{MHz}, \mathrm{CDCl}_{3}\right): \delta \mathrm{ppm} 8.10-8.08$ (d, 2H, $J=7.5 \mathrm{~Hz}$, Ar), 7.58-7.55 (t, 1H, $J=7.5 \mathrm{~Hz}, \mathrm{Ar}), 7.46-7.42$ (t, 2H, $J=7.5 \mathrm{~Hz}, \mathrm{Ar}), 5.10$ (s, 2H, -CH2), 2.08(br-s, $3 \mathrm{H}$, adamantane- $\mathrm{CH}$ ), 1.94-1.93 (br-d, 6H, adamantane- $\mathrm{CH}_{2}$ ), 1.80-1.72 (br-q, 6H, adamantane- $\mathrm{CH}_{2}$ ); ${ }^{13} \mathrm{C}-\mathrm{NMR}\left(125 \mathrm{MHz}, \mathrm{CDCl}_{3}\right): \delta$ ppm $207.16(\mathrm{C}=\mathrm{O}), 166.04(\mathrm{O}-\mathrm{C}=\mathrm{O}), 133.19,129.87,129.58,128.37$ (Ar), 64.94 (-CH2), 45.39, 38.02, 36.47, 27.79 (adamantane-Cs).

2-(Adamantan-1-yl)-2-oxoethyl 2-chlorobenzoate (2b): Solvent for growing crystal: acetone and ethanol (1: 1 v/v); yield: 83\%; M.P. 348-350 K; FTIR (ATR (solid) $\left.\mathrm{cm}^{-1}\right)$ : 2904, $2850(\mathrm{C}-\mathrm{H}, v), 1709(\mathrm{C}=\mathrm{O}, v)$, 1590, $1416(\mathrm{Ar}, \mathrm{C}=\mathrm{C}, v), 1247,1024(\mathrm{C}-\mathrm{O}, v), 742(\mathrm{C}-\mathrm{Cl}, v) ;{ }^{1} \mathrm{H}-\mathrm{NMR}\left(500 \mathrm{MHz}, \mathrm{CDCl}_{3}\right): \delta \mathrm{ppm}$ 8.01-8.00 (d, 1H, $J=8.0 \mathrm{~Hz}, \mathrm{Ar}), 7.47-7.43$ (q, 2H, $J=8.0 \mathrm{~Hz}, \mathrm{Ar}), 7.35-7.32$ (t, 1H, $J=8.0 \mathrm{~Hz}, \mathrm{Ar})$, $5.12\left(\mathrm{~s}, 2 \mathrm{H},-\mathrm{CH}_{2}\right), 2.09$ (br-s, 3H, adamantane-CH), 1.93-1.93 (br-d, 6H, adamantane- $\left.\mathrm{CH}_{2}\right), 1.80-1.72$ (br-q, 6H, adamantane- $\left.\mathrm{CH}_{2}\right) ;{ }^{13} \mathrm{C}-\mathrm{NMR}\left(125 \mathrm{MHz}, \mathrm{CDCl}_{3}\right): \delta$ ppm $206.98(\mathrm{C}=\mathrm{O}), 164.89(\mathrm{O}-\mathrm{C}=\mathrm{O}), 133.98$, 132.84, 131.95, 131.03, 129.35, 126.62 (Ar), 65.18(- $\left.\mathrm{CH}_{2}\right), 45.38$ 37.99, 36.44, 27.75 (adamantane-Cs).

2-(Adamantan-1-yl)-2-oxoethyl 3-chlorobenzoate (2c): Solvent for growing crystal: acetone, ethanol and acetonitrile (1:1:1 v/v/v); yield: 73\%; M.P. 403-405 K; FTIR (ATR (solid) $\left.\mathrm{cm}^{-1}\right)$ : 2911, $2850(\mathrm{C}-\mathrm{H}, v)$, 
$1718(\mathrm{C}=\mathrm{O}, v), 1571,1442(\mathrm{Ar}, \mathrm{C}=\mathrm{C}, v), 1295,1253,1130(\mathrm{C}-\mathrm{O}, v), 745(\mathrm{C}-\mathrm{Cl}, \mathrm{v}) ;{ }^{1} \mathrm{H}-\mathrm{NMR}(500 \mathrm{MHz}$, $\mathrm{CDCl}_{3}$ ): $\delta$ ppm 8.07 (s, 1H, Ar), 7.98-7.96 (d, 1H, J=8.0 Hz, Ar), 7.55-7.54 (d, 1H, J=8.0 Hz, Ar), 7.41-7.38 (t, 1H, J=8.0 Hz, Ar), 5.11 (s, 2H, - $\mathrm{CH}_{2}$ ), 2.09 (br-s, 3H, adamantane-CH), 1.93-1.93 (br-d, $6 \mathrm{H}$, adamantane- $\mathrm{CH}_{2}$ ), $1.800-1.719$ (br-q, $6 \mathrm{H}$, adamantane- $\left.\mathrm{CH}_{2}\right) ;{ }^{13} \mathrm{C}-\mathrm{NMR}\left(125 \mathrm{MHz}, \mathrm{CDCl}_{3}\right): \delta \mathrm{ppm}$ $206.94(\mathrm{C}=\mathrm{O}), 164.88(\mathrm{O}-\mathrm{C}=\mathrm{O}), 134.56,133.27,131.27,129.95,129.73,128.01(\mathrm{Ar}), 65.23\left(-\mathrm{CH}_{2}\right), 45.37$, $37.98,36.43,27.74$ (adamantane-Cs).

2-(Adamantan-1-yl)-2-oxoethyl 4-chlorobenzoate (2d): Solvent for growing crystal: acetone, ethanol and acetonitrile (1:1:1 v/v/v); yield: 75\%; M.P. 411-413 K; FTIR (ATR (solid) $\left.\mathrm{cm}^{-1}\right): 2910,2851(\mathrm{C}-\mathrm{H}, v)$, $1723(\mathrm{C}=\mathrm{O}, v), 1593,1421$ (Ar, C=C, v), 1269, $1119(\mathrm{C}-\mathrm{O}, v), 752(\mathrm{C}-\mathrm{Cl}, v) ;{ }^{1} \mathrm{H}-\mathrm{NMR}(500 \mathrm{MHz}$, $\left.\mathrm{CDCl}_{3}\right): \delta \mathrm{ppm} 8.03-8.01(\mathrm{~d}, 2 \mathrm{H}, J=8.8 \mathrm{~Hz}, \mathrm{Ar}), 7.43-7.41(\mathrm{~d}, 2 \mathrm{H}, J=8.8 \mathrm{~Hz}, \mathrm{Ar}), 5.09$ (s, 2H, $-\mathrm{CH}_{2}$ ), 2.08 (br-s, 3H, adamantane-CH), 1.93-1.92 (br-d, 6H, adamantane- $\mathrm{CH}_{2}$ ), 1.80-1.72 (br-q, 6H, adamantane- $\left.\mathrm{CH}_{2}\right) ;{ }^{13} \mathrm{C}-\mathrm{NMR}\left(125 \mathrm{MHz}, \mathrm{CDCl}_{3}\right)$ : $\delta \mathrm{ppm} 207.07(\mathrm{C}=\mathrm{O}), 165.19(\mathrm{O}-\mathrm{C}=\mathrm{O}), 139.71,131.27$, 128.76, 128.01 (Ar), 65.11(- $\left.\mathrm{CH}_{2}\right), 45.37,37.99,36.43,27.75$ (adamantane-Cs).

2-(Adamantan-1-yl)-2-oxoethyl 2,4-dichlorobenzoate (2e): Solvent for growing crystal: acetone; yield: 85\%; M.P. 395-397 K; FTIR (ATR (solid) cm $\mathrm{cm}^{-1}$ ): 2912, 2850 (C-H, v), 1711 (C=O, v), 1583, 1417 $(\mathrm{Ar}, \mathrm{C}=\mathrm{C}, v), 1244,1129,1098,1023(\mathrm{C}-\mathrm{O}, v), 829(\mathrm{C}-\mathrm{Cl}, v) ;{ }^{1} \mathrm{H}-\mathrm{NMR}\left(500 \mathrm{MHz}, \mathrm{CDCl}_{3}\right): \delta \mathrm{ppm}$ 7.98-7.96 (d, 1H, $J=8.5 \mathrm{~Hz}, \mathrm{Ar}), 7.48(\mathrm{~s}, 1 \mathrm{H}, \mathrm{Ar}), 7.33-7.31$ (d, 1H, $J=8.5 \mathrm{~Hz}, \mathrm{Ar}), 5.11$ (s, 2H, $-\mathrm{CH}_{2}$ ), 2.09 (br-s, 3H, adamantane-CH), 1.93-1.92 (br-d, 6H, adamantane- $\mathrm{CH}_{2}$ ), 1.80-1.72 (br-q, 6H, adamantane- $\left.\mathrm{CH}_{2}\right) ;{ }^{13} \mathrm{C}-\mathrm{NMR}\left(125 \mathrm{MHz}, \mathrm{CDCl}_{3}\right): \delta \mathrm{ppm} 206.86(\mathrm{C}=\mathrm{O}), 164.01(\mathrm{O}-\mathrm{C}=\mathrm{O}), 138.66$, 135.20, 133.00, 131.00, 127.62, 127.06 (Ar), 65.30(- $\left.\mathrm{CH}_{2}\right), 45.38,37.98,36.42,27.73$ (adamantane-Cs).

2-(Adamantan-1-yl)-2-oxoethyl 2-methylbenzoate (2f): Solvent for growing crystal: acetone; yield: 83\%; M.P. 367-369 K; FTIR (ATR (solid) $\mathrm{cm}^{-1}$ ): 2905, 2851 (C-H, v), 1708 (C=O, v), 1602, 1415 $(\mathrm{Ar}, \mathrm{C}=\mathrm{C}, v), 1253,1026(\mathrm{C}-\mathrm{O}, v), 733(\mathrm{C}-\mathrm{H}, \omega) ;{ }^{1} \mathrm{H}-\mathrm{NMR}\left(500 \mathrm{MHz}, \mathrm{CDCl}_{3}\right): \delta \mathrm{ppm} 8.02-8.00(\mathrm{~d}$, $1 \mathrm{H}, J=8.0 \mathrm{~Hz}, \mathrm{Ar}), 7.42-7.39$ (t, $1 \mathrm{H}, J=8.0 \mathrm{~Hz}, \mathrm{Ar}), 7.25-7.24$ (m, 2H, Ar), 5.09 (s, 2H, $-\mathrm{CH}_{2}$ ), 2.09 (br-s, 3H, adamantane-CH), 1.94-1.94 (br-d, 6H, adamantane- $\mathrm{CH}_{2}$ ), 1.80-1.72 (br-q, 6H, adamantane- $\left.\mathrm{CH}_{2}\right) ;{ }^{13} \mathrm{C}-\mathrm{NMR}\left(125 \mathrm{MHz}, \mathrm{CDCl}_{3}\right): \delta \mathrm{ppm} 207.41(\mathrm{C}=\mathrm{O}), 166.98(\mathrm{O}-\mathrm{C}=\mathrm{O}), 140.43$, 132.19, 131.58, 130.89, 129.06, $125.72(\mathrm{Ar}), 64.74\left(-\mathrm{CH}_{2}\right), 45.37,38.03,36.47,27.78$ (adamantane-Cs), $21.60\left(-\mathrm{CH}_{3}\right)$.

2-(Adamantan-1-yl)-2-oxoethyl 3-methylbenzoate (2g): Solvent for growing crystal: acetone, ethanol and acetonitrile (1:1:1 v/v/v); yield: 75\%; M.P. 372-374 K; FTIR (ATR (solid) $\left.\mathrm{cm}^{-1}\right)$ : 2902, $2848(\mathrm{C}-\mathrm{H}, \mathrm{v}$ ), $1724(\mathrm{C}=\mathrm{O}, v), 1588,1421,(\mathrm{Ar}, \mathrm{C}=\mathrm{C}, \mathrm{v}), 1302,1279,1195,1109(\mathrm{C}-\mathrm{O}, \mathrm{v}), 740(\mathrm{C}-\mathrm{H}, \omega)$; ${ }^{1} \mathrm{H}-\mathrm{NMR}$ $\left(500 \mathrm{MHz}, \mathrm{CDCl}_{3}\right): \delta$ ppm 7.91 (s, 1H, Ar), 7.89-7.88 (d, 2H, $\left.J=7.5 \mathrm{~Hz}, \mathrm{Ar}\right), 7.39-7.37$ (d, 1H, $J=7.5 \mathrm{~Hz}, \mathrm{Ar}$ ), 7.35-7.32 (t, 1H, $J=7.5 \mathrm{~Hz} \mathrm{Ar}$ ), 5.09 (s, 2H, - $\mathrm{CH}_{2}$ ), 2.08 (br-s, 3H, adamantane-CH), 1.94-1.93 (br-d, 6H, adamantane- $\mathrm{CH}_{2}$ ), $1.80-1.72$ (br-q, 6H, adamantane- $\left.\mathrm{CH}_{2}\right)$; ${ }^{13} \mathrm{C}-\mathrm{NMR}(125 \mathrm{MHz}$, $\left.\mathrm{CDCl}_{3}\right): \delta$ ppm $207.32(\mathrm{C}=\mathrm{O}), 166.25(\mathrm{O}-\mathrm{C}=\mathrm{O}), 138.17,134.00,130.39,129.44,128.29,127.04(\mathrm{Ar})$, $64.91\left(-\mathrm{CH}_{2}\right), 45.39,38.01,36.47,27.78$ (adamantane-Cs), $21.26\left(-\mathrm{CH}_{3}\right)$.

2-(Adamantan-1-yl)-2-oxoethyl 4-methylbenzoate (2h): Solvent for growing crystal: acetone; yield: 81\%; M.P. 413-415 K; FTIR (ATR (solid) $\mathrm{cm}^{-1}$ ): 2902, 2853 (C-H, v), 1714 (C=O, v), 1610, 1418, (Ar, 
$\mathrm{C}=\mathrm{C}, v), 1258,1115,(\mathrm{C}-\mathrm{O}, v), 747(\mathrm{C}-\mathrm{H}, \omega) ;{ }^{1} \mathrm{H}-\mathrm{NMR}\left(500 \mathrm{MHz}, \mathrm{CDCl}_{3}\right): \delta$ ppm 7.98-7.97 (d, 2H, $J=8.0 \mathrm{~Hz}, \mathrm{Ar}), 7.25-7.23$ (d, 2H, $J=8.0 \mathrm{~Hz}, \mathrm{Ar}), 5.08\left(\mathrm{~s}, 2 \mathrm{H},-\mathrm{CH}_{2}\right), 2.41$ (s, 3H, $\left.-\mathrm{CH}_{3}\right), 2.08$ (br-s, 3H, adamantane- $\mathrm{CH}$ ), 1.93-1.93 (br-d, 6H, adamantane- $\mathrm{CH}_{2}$ ), 1.80-1.72 (br-q, 6H, adamantane- $\mathrm{CH}_{2}$ ); ${ }^{13} \mathrm{C}-\mathrm{NMR}\left(125 \mathrm{MHz}, \mathrm{CDCl}_{3}\right): \delta$ ppm $207.37(\mathrm{C}=\mathrm{O}), 166.12(\mathrm{O}-\mathrm{C}=\mathrm{O}), 143.93,129.92,129.10,126.80$ (Ar), $64.82\left(-\mathrm{CH}_{2}\right), 45.39,38.01,36.47,27.79$ (adamantane-Cs), $21.70\left(-\mathrm{CH}_{3}\right)$.

2-(Adamantan-1-yl)-2-oxoethyl 2-methoxybenzoate (2i): Solvent for growing crystal: acetone and ethanol (1: 1 v/v); yield: 80\%; M.P. 362-364 K; FTIR (ATR (solid) $\left.\mathrm{cm}^{-1}\right)$ : 2902, $2851(\mathrm{C}-\mathrm{H}, v), 1702(\mathrm{C}=\mathrm{O}, v)$, 1599, 1442 (Ar, C=C, v), 1248, 1096, $1020(\mathrm{C}-\mathrm{O}, v), 758(\mathrm{C}-\mathrm{H}, \omega)$; ${ }^{1} \mathrm{H}-\mathrm{NMR}\left(500 \mathrm{MHz}, \mathrm{CDCl}_{3}\right): \delta \mathrm{ppm}$ 7.97-7.95 (d, 1H, $J=8.5 \mathrm{~Hz}, \mathrm{Ar}), 7.50-7.47$ (t, 1H, $J=8.5 \mathrm{~Hz}, \mathrm{Ar}), 7.01-6.97$ (t, 2H, $J=8.5 \mathrm{~Hz}, \mathrm{Ar}$ ), 5.07 (s, $\left.2 \mathrm{H},-\mathrm{CH}_{2}\right), 3.91$ (s, 3H, - $\mathrm{CH}_{3}$ ), 2.07 (br-s, 3H, adamantane- $\mathrm{CH}$ ), 1.93-1.93 (br-d, 6H, adamantane- $\mathrm{CH}_{2}$ ), 1.79-1.71 (br-q, 6H, adamantane- $\left.\mathrm{CH}_{2}\right)$; ${ }^{13} \mathrm{C}-\mathrm{NMR}\left(125 \mathrm{MHz}, \mathrm{CDCl}_{3}\right)$ : $\delta$ ppm $207.41(\mathrm{C}=\mathrm{O}), 165.23$ $(\mathrm{O}-\mathrm{C}=\mathrm{O}), 159.52,133.90,132.21,120.17,119.19,111.99(\mathrm{Ar}), 64.72\left(-\mathrm{CH}_{2}\right), 56.03\left(-\mathrm{CH}_{3}\right), 45.38$, $38.01,36.48,27.79$ (adamantane-Cs).

2-(Adamantan-1-yl)-2-oxoethyl 3-methoxybenzoate (2j): Solvent for growing crystal: acetone and ethanol (1: 1 v/v); Yield: 74\%; M.P. 430-432 K; FTIR (ATR (solid) $\left.\mathrm{cm}^{-1}\right)$ : 2926, $2853(\mathrm{C}-\mathrm{H}, v), 1711(\mathrm{C}=\mathrm{O}, v)$, 1584, 1489 (Ar, C=C, v), 1288, 1221, 1029 (C-O, v), 759 (C-H, $\omega) .{ }^{1} \mathrm{H}-\mathrm{NMR}\left(500 \mathrm{MHz}, \mathrm{CDCl}_{3}\right)$ : $\delta$ ppm 7.70-7.68 (d, 1H, $J=8.0 \mathrm{~Hz}, \mathrm{Ar}), 7.60$ (s, 1H, Ar), 7.37-7.33 (t, 1H, $J=8.0 \mathrm{~Hz}, \mathrm{Ar}$ ), 7.13-7.11 (m, 1H, Ar), 5.09 (s, 2H, - $\mathrm{CH}_{2}$ ), 3.85 (s, 3H, - $\mathrm{CH}_{3}$ ), 2.08 (br-s, 3H, adamantane-CH), 1.94-1.93 (br-d, $6 \mathrm{H}$, adamantane- $\left.\mathrm{CH}_{2}\right), 1.80-1.72$ (br-q, $6 \mathrm{H}$, adamantane- $\left.\mathrm{CH}_{2}\right) ;{ }^{13} \mathrm{C}-\mathrm{NMR}\left(125 \mathrm{MHz}, \mathrm{CDCl}_{3}\right): \delta \mathrm{ppm}$ $207.18(\mathrm{C}=\mathrm{O}), 165.96(\mathrm{O}-\mathrm{C}=\mathrm{O}), 159.56,130.79,129.42,122.35,120.04,114.05(\mathrm{Ar}), 65.04\left(-\mathrm{CH}_{2}\right)$, $55.44\left(-\mathrm{CH}_{3}\right), 45.39,38.01,36.46,27.77$ (adamantane-Cs).

2-(Adamantan-1-yl)-2-oxoethyl 4-methoxybenzoate (2k): Solvent for growing crystal: acetone and ethanol (1: $1 v / v$ ); yield: 80\%; M.P. 390-392 K; FTIR (ATR (solid) $\mathrm{cm}^{-1}$ ): 2903, 2851 (C-H, v), 1710 $(\mathrm{C}=\mathrm{O}, v), 1605,1417(\mathrm{Ar}, \mathrm{C}=\mathrm{C}, \mathrm{v})$, 1253, 1167, $1028(\mathrm{C}-\mathrm{O}, v), 767(\mathrm{C}-\mathrm{H}, \omega) ;{ }^{1} \mathrm{H}-\mathrm{NMR}(500 \mathrm{MHz}$, $\left.\mathrm{CDCl}_{3}\right): \delta$ ppm 8.05-8.04 (d, 2H, $\left.J=9.0 \mathrm{~Hz}, \mathrm{Ar}\right), 6.93-6.91(\mathrm{~d}, 2 \mathrm{H}, J=9.0 \mathrm{~Hz} \mathrm{Ar}), 5.07$ (s, 2H, $-\mathrm{CH}_{2}$ ), 3.86 (s, 3H, - $\mathrm{CH}_{3}$ ), 2.08 (br-s, 3H, adamantane- $\mathrm{CH}$ ), 1.93-1.93 (br-d, 6H, adamantane- $\mathrm{CH}_{2}$ ), 1.80-1.72 (br-q, 6H, adamantane-CH$)$; ${ }^{13} \mathrm{C}-\mathrm{NMR}\left(125 \mathrm{MHz}, \mathrm{CDCl}_{3}\right): \delta$ ppm $207.54(\mathrm{C}=\mathrm{O}), 165.77$ $(\mathrm{O}-\mathrm{C}=\mathrm{O}), 163.61,131.96,121.94,113.66(\mathrm{Ar}), 64.73\left(-\mathrm{CH}_{2}\right), 55.44\left(-\mathrm{CH}_{3}\right), 45.39,38.02,36.47$, 27.79 (adamantane-Cs).

2-(Adamantan-1-yl)-2-oxoethyl 2-nitrobenzoate (2l): Solvent for growing crystal: acetone, ethanol and acetonitrile (1:1:1 v/v/v); yield: 70\%; M.P. 391-393 K; FTIR (ATR (solid) $\left.\mathrm{cm}^{-1}\right): 2919,2850(\mathrm{C}-\mathrm{H}, v)$, $1731(\mathrm{C}=\mathrm{O}, v), 1579,1450(\mathrm{Ar}, \mathrm{C}=\mathrm{C}, v), 1535,1353(\mathrm{~N}-\mathrm{O}, v), 1290,1132,1079(\mathrm{C}-\mathrm{O}, v), 732(\mathrm{C}-\mathrm{H}, \omega)$; ${ }^{1} \mathrm{H}-\mathrm{NMR}\left(500 \mathrm{MHz}, \mathrm{CDCl}_{3}\right)$ : $\delta \mathrm{ppm} 7.97-7.95(\mathrm{~d}, 1 \mathrm{H}, J=7.5 \mathrm{~Hz}, \mathrm{Ar}), 7.94-7.92(\mathrm{~d}, 1 \mathrm{H}, J=7.5 \mathrm{~Hz} \mathrm{Ar})$, 7.73-7.70 (t, 1H, $J=7.5 \mathrm{~Hz}, \mathrm{Ar}), 7.66-7.63$ (t, 1H, $J=7.5 \mathrm{~Hz}, \mathrm{Ar}), 5.12$ (s, 2H, -CH2), 2.09 (br-s, 3H, adamantane- $\mathrm{CH}$ ), $1.92\left(\mathrm{br}-\mathrm{s}, 6 \mathrm{H}\right.$, adamantane- $\left.\mathrm{CH}_{2}\right), 1.78-1.74$ (br-d, $6 \mathrm{H}$, adamantane- $\left.\mathrm{CH}_{2}\right) ;{ }^{13} \mathrm{C}-\mathrm{NMR}$ (125 MHz, $\left.\mathrm{CDCl}_{3}\right): \delta$ ppm $206.75(\mathrm{C}=\mathrm{O}), 164.97(\mathrm{O}-\mathrm{C}=\mathrm{O}), 147.74,133.17,131.78,130.35,127.46$, 123.94 (Ar), $65.92\left(-\mathrm{CH}_{2}\right), 45.39,37.95,36.41,27.72$ (adamantane-Cs). 
2-(Adamantan-1-yl)-2-oxoethyl 3-nitrobenzoate (2m): yield: 72\%; M.P. 385-387 K; FTIR (ATR (solid) $\mathrm{cm}^{-1}$ ): 3092 (Ar, C-H, v), 2917, $2851(\mathrm{C}-\mathrm{H}, v), 1727$ (C=O, v), 1631, 1421 (Ar, C=C, v), 1533, $1347(\mathrm{~N}-\mathrm{O}, v), 1297,1261,1131(\mathrm{C}-\mathrm{O}, \mathrm{v}), 716(\mathrm{C}-\mathrm{H}, \omega) ;{ }^{1} \mathrm{H}-\mathrm{NMR}\left(500 \mathrm{MHz}, \mathrm{CDCl}_{3}\right): \delta$ ppm 8.92 (s, 1H, Ar), 8.45-8.43 (d, 1H, $J=8.1 \mathrm{~Hz}, \mathrm{Ar}), 8.42-8.40$ (d, 1H, $J=8.1 \mathrm{~Hz}, \mathrm{Ar}), 7.69-7.65$ (t, 1H, $J=8.1 \mathrm{~Hz}, \mathrm{Ar}), 5.17$ (s, 2H, - $\mathrm{CH}_{2}$ ), 2.10 (br-s, 3H, adamantane-CH), 1.94 (br-s, 6H, adamantane- $\mathrm{CH}_{2}$ ), 1.79-1.76 (br-d, 6H, adamantane- $\left.\mathrm{CH}_{2}\right)$; ${ }^{13} \mathrm{C}-\mathrm{NMR}\left(125 \mathrm{MHz}, \mathrm{CDCl}_{3}\right): \delta$ ppm $206.60(\mathrm{C}=\mathrm{O}), 164.00$ $(\mathrm{O}-\mathrm{C}=\mathrm{O}), 148.31,135.52,131.35,129.67,127.66,124.92(\mathrm{Ar}), 65.61\left(-\mathrm{CH}_{2}\right), 45.39,37.99,36.42$, 27.74 (adamantane-Cs).

2-(Adamantan-1-yl)-2-oxoethyl 4-nitrobenzoate (2n): Solvent for growing crystal: acetone, ethanol and acetonitrile (1:1:1 v/v/v); yield: 76\%; M.P. 437-439 K; FTIR (ATR (solid) $\mathrm{cm}^{-1}$ ): 3112 (Ar, C-H, v), 2905, $2854(\mathrm{C}-\mathrm{H}, v), 1723(\mathrm{C}=\mathrm{O}, v), 1606,1422(\mathrm{Ar}, \mathrm{C}=\mathrm{C}, v), 1530,1344(\mathrm{~N}-\mathrm{O}, v), 1283,1118$ $(\mathrm{C}-\mathrm{O}, v), 714(\mathrm{C}-\mathrm{H}, \omega)$; ${ }^{1} \mathrm{H}-\mathrm{NMR}\left(500 \mathrm{MHz}, \mathrm{CDCl}_{3}\right): \delta \mathrm{ppm} 8.31-8.29(\mathrm{~d}, 2 \mathrm{H}, J=8.5 \mathrm{~Hz}, \mathrm{Ar})$, 8.27-8.25 (d, 2H, $J=8.5 \mathrm{~Hz} \mathrm{Ar}$ ), 5.16 (s, 2H, - $\mathrm{CH}_{2}$ ), 2.10 (br-s, 3H, adamantane-CH), 1.94 (br-s, 6H, adamantane- $\mathrm{CH}_{2}$ ), $1.81-1.73$ (br-q, 6H, adamantane- $\left.\mathrm{CH}_{2}\right) ;{ }^{13} \mathrm{C}-\mathrm{NMR}\left(125 \mathrm{MHz}, \mathrm{CDCl}_{3}\right): \delta \mathrm{ppm}$ $206.61(\mathrm{C}=\mathrm{O}), 164.21(\mathrm{O}-\mathrm{C}=\mathrm{O}), 150.72,134.97,131.00,123.56(\mathrm{Ar}), 65.61\left(-\mathrm{CH}_{2}\right), 45.40,37.99$, $36.41,27.73$ (adamantane-Cs).

2-(Adamantan-1-yl)-2-oxoethyl 2-aminobenzoate (2o): Solvent for growing crystal: acetone, ethanol and acetonitrile (1:1:1 v/v); yield: 76\%; M.P. 440-442 K; FTIR (ATR (solid) $\mathrm{cm}^{-1}$ ): 3497, $3373(\mathrm{~N}-\mathrm{H}, v$ ), 2902, $2848(\mathrm{C}-\mathrm{H}, v), 1695(\mathrm{C}=\mathrm{O}, v), 1613,1418(\mathrm{Ar}, \mathrm{C}=\mathrm{C}, v), 1581(\mathrm{~N}-\mathrm{H}, \delta), 1243,1104(\mathrm{C}-\mathrm{O}, v)$, $746(\mathrm{C}-\mathrm{H}, \omega)$; ${ }^{1} \mathrm{H}-\mathrm{NMR}\left(500 \mathrm{MHz}, \mathrm{CDCl}_{3}\right): \delta \mathrm{ppm} 7.95-7.94(\mathrm{~d}, 1 \mathrm{H}, J=8.3 \mathrm{~Hz}, \mathrm{Ar}), 7.29-7.26$ (t, 1H, $J=8.3 \mathrm{~Hz} \mathrm{Ar}$ ), 6.67-6.64 (m, 2H, Ar), 5.07 (s, 2H, - $\left.\mathrm{CH}_{2}\right), 2.08$ (br-s, 3H, adamantane-CH), 1.93 (br-s, 6H, adamantane- $\mathrm{CH}_{2}$ ), $1.80-1.72$ (br-d, 6H, adamantane- $\left.\mathrm{CH}_{2}\right) ;{ }^{13} \mathrm{C}-\mathrm{NMR}(125 \mathrm{MHz}$, $\left.\mathrm{CDCl}_{3}\right): \delta$ ppm $207.64(\mathrm{C}=\mathrm{O}), 167.29(\mathrm{O}-\mathrm{C}=\mathrm{O}), 150.52,134.38,131.63,116.67,116.41,110.34(\mathrm{Ar})$, $64.46\left(-\mathrm{CH}_{2}\right), 45.38,38.05,36.47,27.79$ (adamantane-Cs).

2-(Adamantan-1-yl)-2-oxoethyl 3-aminobenzoate (2p): Solvent for growing crystal: acetone, ethanol and acetonitrile $(1: 1: 1 \mathrm{v} / \mathrm{v} / \mathrm{v})$; yield: 70\%; M.P. 396-398 K; FTIR (ATR (solid) cm $\left.{ }^{-1}\right): 3465,3347$ $(\mathrm{N}-\mathrm{H}, v), 2902,2849(\mathrm{C}-\mathrm{H}, \mathrm{v}), 1700(\mathrm{C}=\mathrm{O}, \mathrm{v}), 1631,1461(\mathrm{Ar}, \mathrm{C}=\mathrm{C}, \mathrm{v}), 1603(\mathrm{~N}-\mathrm{H}, \delta), 1248,1113$ $(\mathrm{C}-\mathrm{O}, v), 751(\mathrm{C}-\mathrm{H}, \omega) ;{ }^{1} \mathrm{H}-\mathrm{NMR}\left(500 \mathrm{MHz}, \mathrm{CDCl}_{3}\right)$ : $\delta \mathrm{ppm} 7.50-7.49(\mathrm{~d}, 1 \mathrm{H}, J=7.9 \mathrm{~Hz}, \mathrm{Ar}), 7.42$ (s, 1H, Ar), 7.24-7.21 (t, 1H, $J=7.9 \mathrm{~Hz}, \mathrm{Ar}), 6.91-6.89$ (d, 1H, $J=7.9 \mathrm{~Hz}, \mathrm{Ar}), 5.08\left(\mathrm{~s}, 2 \mathrm{H},-\mathrm{CH}_{2}\right)$, 2.08 (br-s, 3H, adamantane- $\mathrm{CH}$ ), 1.93-1.92 (br-d, 6H, adamantane- $\mathrm{CH}_{2}$ ), 1.79-1.72 (br-q, 6H, adamantane- $\left.\mathrm{CH}_{2}\right) ;{ }^{13} \mathrm{C}-\mathrm{NMR}\left(125 \mathrm{MHz}, \mathrm{CDCl}_{3}\right): \delta \mathrm{ppm} 207.36(\mathrm{C}=\mathrm{O}), 166.17(\mathrm{O}-\mathrm{C}=\mathrm{O}), 145.98$, 130.49, 129.34, 120.42, 119.99, 116.28 (Ar), $64.91\left(-\mathrm{CH}_{2}\right), 45.39$, 38.00, 36.46, 27.77(adamantane-Cs).

2-(Adamantan-1-yl)-2-oxoethyl 4-aminobenzoate (2q): yield: 76\%; M.P. 428-430 K; FTIR (ATR (solid) $\left.\mathrm{cm}^{-1}\right)$ : 3493, $3362(\mathrm{~N}-\mathrm{H}, \mathrm{v}), 2906,2850(\mathrm{C}-\mathrm{H}, \mathrm{v}), 1697(\mathrm{C}=\mathrm{O}, \mathrm{v}), 1619,1414(\mathrm{Ar}, \mathrm{C}=\mathrm{C}, v)$, $1600(\mathrm{~N}-\mathrm{H}, \delta), 1276,1118(\mathrm{C}-\mathrm{O}, \mathrm{v}), 768(\mathrm{C}-\mathrm{H}, \omega) ;{ }^{1} \mathrm{H}-\mathrm{NMR}\left(500 \mathrm{MHz}, \mathrm{CDCl}_{3}\right)$ : $\delta$ ppm 7.91-7.89 (d, 2H, $J=8.5 \mathrm{~Hz}, \mathrm{Ar}$ ), 6.65-6.63 (d, 2H, $J=8.5 \mathrm{~Hz}, \mathrm{Ar}$ ), 5.04 (s, 2H, -CH2), 2.07 (br-s, 3H, adamantane-CH), 1.93-1.92 (br-d, 6H, adamantane- $\mathrm{CH}_{2}$ ), 1.79-1.71 (br-q, 6H, adamantane- $\mathrm{CH}_{2}$ ); ${ }^{13} \mathrm{C}-\mathrm{NMR}\left(125 \mathrm{MHz}, \mathrm{CDCl}_{3}\right): \delta$ ppm $207.90(\mathrm{C}=\mathrm{O}), 166.03(\mathrm{O}-\mathrm{C}=\mathrm{O}), 151.08,132.00,119.04,113.92$ (Ar), $64.50\left(-\mathrm{CH}_{2}\right), 45.57,38.02,36.60,27.77$ (adamantane-Cs). 
2-(Adamantan-1-yl)-2-oxoethyl 2-pyridinecarboxylate (2r): Solvent for growing crystal: acetone, ethanol and acetonitrile $(1: 1: 1 \mathrm{v} / v / v)$; yield: 76\%; M.P. 402-404 K; FTIR (ATR (solid) $\mathrm{cm}^{-1}$ ): 2903, $2849(\mathrm{C}-\mathrm{H}, \mathrm{v}), 1707(\mathrm{C}=\mathrm{O}, \mathrm{v}), 1582,1419$ (Ar, C=C, v), $1304(\mathrm{C}-\mathrm{N}, \mathrm{v})$ 1242, 1128 (C-O, v), $745(\mathrm{C}-\mathrm{H}$, $\omega)$ ) ${ }^{1} \mathrm{H}-\mathrm{NMR}\left(500 \mathrm{MHz}, \mathrm{CDCl}_{3}\right): \delta \mathrm{ppm} 8.79-8.78(\mathrm{~d}, 1 \mathrm{H}, J=4.7 \mathrm{~Hz}, \mathrm{Ar}), 8.19-8.17(\mathrm{~d}, 1 \mathrm{H}, J=4.7 \mathrm{~Hz}$, Ar), 7.88-7.86 (t, 1H, $J=4.7 \mathrm{~Hz}, \mathrm{Ar}), 7.52-7.50$ (q, 1H, $J=4.7 \mathrm{~Hz}, \mathrm{Ar}$ ), 5.20 (s, 2H, -CH2), 2.09 (br-s, $3 \mathrm{H}$, adamantane- $\mathrm{CH}$ ), 1.93-1.93 (br-d, 6H, adamantane- $\mathrm{CH}_{2}$ ), 1.80-1.72 (br-q, 6H, adamantane- $\mathrm{CH}_{2}$ ); ${ }^{13} \mathrm{C}-\mathrm{NMR}\left(125 \mathrm{MHz}, \mathrm{CDCl}_{3}\right): \delta$ ppm $206.36(\mathrm{C}=\mathrm{O}), 164.54(\mathrm{O}-\mathrm{C}=\mathrm{O}), 149.86,147.41,137.12,127.18$, 125.53 (Ar), $65.70\left(-\mathrm{CH}_{2}\right), 45.31,37.99,36.44,27.75$ (adamantane-Cs).

\subsection{Bioactivity Methods}

\subsubsection{Hydrogen Peroxide Radical Scavenging Assay}

The abilities of adamantyl-based compounds to scavenge hydroxyl radical were determined by following the method of [50] with some modifications. The solution of hydrogen peroxide (40 $\mathrm{mM})$ was prepared in PBS (phosphate-buffered saline). The adamantyl-based derivatives with a concentration of $250 \mu \mathrm{g} / \mathrm{mL}$ were prepared in PBS and added to $0.6 \mathrm{~mL}$ of hydrogen peroxide solution. The mixture was mixed well and incubated at room temperature for $10 \mathrm{~min}$. The absorbance was determined at $230 \mathrm{~nm}$ by using a spectrophotometer. Ascorbic acid was used as a standard drug, while PBS was used as the blank. The percentage of hydrogen peroxide scavenging activity was calculated by using the following formula:

$$
\text { Percentage of scavenging activity }=\frac{\text { Absorbance of control }- \text { Absorbance of sample }}{\text { Absorbance of control }} \times 100
$$

\subsubsection{DPPH Radical Scavenging Assay}

The DPPH radical scavenging assay was used to evaluate the antioxidant properties of the adamantyl-based compounds. This assay was carried out following the method of [51]. The DPPH solution $(0.16 \mathrm{mM})$ was prepared in ethanol. The adamantyl-based derivatives were prepared in ethanol with concentrations of 250,500 and $1000 \mu \mathrm{g} / \mathrm{mL}$. Approximately $100 \mu \mathrm{L}$ of each adamantyl-based derivative were mixed with $100 \mu \mathrm{L}$ of DPPH solution in a 96-well plate. Each sample and the control were prepared in triplicate. The plate was then incubated in a dark room at room temperature for $30 \mathrm{~min}$, and the absorbance was measured at $517 \mathrm{~nm}$ with a microplate reader. Ascorbic acid was used as a standard drug. The percentage of DPPH free radical scavenging activity was calculated by using the following formula:

$$
\text { Percentage of scavenging activity }=\frac{\text { Absorbance of blank }- \text { Absorbance of sample }}{\text { Absorbance of blank }} \times 100
$$

\subsubsection{Protein Denaturation Assay}

Adamantyl-based compounds were tested on egg albumin to observe their anti-inflammatory activity against protein denaturation. The assay was performed following the method described by [52] with some modifications. The $2.5 \mathrm{~mL}$ of reaction mixture consisted of $0.1 \mathrm{~mL}$ of egg albumin, $1.4 \mathrm{~mL}$ of PBS 
and $1 \mathrm{~mL}$ of adamantyl-based derivatives with a concentration of $250 \mu \mathrm{g} / \mathrm{mL}$. A similar volume of PBS with egg albumin was used as the control. The mixture solutions were incubated at $37{ }^{\circ} \mathrm{C}$ for 15 min and then heat in a water bath at $70{ }^{\circ} \mathrm{C}$ for $5 \mathrm{~min}$. After cooling to room temperature, the absorbance was measured at $660 \mathrm{~nm}$ using a microplate reader. Diclofenac sodium was used as the standard drug. The percentage of inhibition of protein denaturation was calculated by using the following formula:

$$
\text { Percentage of scavenging activity }=\frac{\text { Absorbance of control }- \text { Absorbance of sample }}{\text { Absorbance of control }} \times 100
$$

\section{Conclusions}

A series of 2-(adamantan-1-yl)-2-oxoethyl benzoates, 2(a-q), and 2-(adamantan-1-yl)-2-oxoethyl 2-pyridinecarboxylate, 2r, were synthesized and structurally characterized by FTIR, NMR and single-crystal X-ray diffraction analysis. Introduction of the adamantane moiety into the synthesis of 2-oxopropyl benzoate derivatives produced all molecular structures in synclinal conformation. In crystals, molecules are commonly packed in a head-to-tail (adamantane moiety to phenyl moiety) pattern. These similarities lead to redundant 2D or 3D structural similarity in adamantane-based ester derivatives. The adamantyl-based compounds show selective antioxidant abilities and good hydrogen peroxide radical scavenging activities, especially 2-(adamantan-1-yl)-2-oxoethyl 2-chlorobenzoate, which outperformed the standard compound. Besides that, three nitrogen-containing adamantane compounds, $\mathbf{2 p}, \mathbf{2 q}$ and $\mathbf{2 r}$, show strong anti-inflammatory effects towards protein denaturation, which performed better than diclofenac sodium. Thus, further modifications of adamantane compounds with nitrogen-containing groups can potentially produce promising anti-inflammatory agents for clinical use in the future.

\section{Supplementary Materials}

Supplementary materials can be accessed at: http:/www.mdpi.com/1420-3049/20/10/18827/s1.

\section{Acknowledgments}

The authors thank the Malaysian Government and Universiti Sains Malaysia (USM) for the Research University Individual Grant (1001/PFIZIK/811278). CSCK thanks USM for a postdoctoral research fellowship (2013-2015). HCK and WSL thank the Malaysian Government for the MyBrain15 (MyPhD) scholarship.

\section{Author Contribution}

C.S.C.K. conceived of and designed the experiments. C.S.C.K. and H.C.K. performed the experiments. C.S.C.K., H.C.K., T.S.C., W.S.L., C.K.Q., G.K.L., S.C. and H.K.F. analyzed the data. S.H.M. performed the bioassays (antioxidant and anti-inflammatory). C.K.Q. contributed reagents/ materials/analysis tools. All authors wrote the paper. All authors read and approved the final manuscript.

\section{Conflicts of Interest}

The authors declare no conflict of interest. 


\section{References}

1. Piérard, G.E.; Piérard-Franchimont, C.; Paquet, P.; Quatresooz, P. Spotlight on adapalene. Expert Opin. Drug Metab. Toxicol. 2009, 5, 1565-1575.

2. Reisberg, B.; Doody, R.; Stöffler, A.; Schmitt, F.; Ferris, S.; Möbius, H.J. Memantine in moderate-to-severe alzheimer's disease. N. Engl. J. Med. 2003, 348, 1333-1341.

3. Rosenthal, K.S.; Sokol, M.S.; Ingram, R.L.; Subramanian, R.; Fort, R.C. Tromantadine: Inhibitor of early and late events in herpes simplex virus replication. Antimicrob. Agents Chemother. 1982, 22, 1031-1036.

4. Kelly, J.M.; Miles, M.A.; Skinner, A.C. The anti-influenza virus drug rimantadine has trypanocidal activity. Antimicrob. Agents Chemother. 1999, 43, 985-987.

5. De Clercq, E. Antiviral agents active against influenza a viruses. Nat. Rev. Drug Discov. 2006, 5, 1015-1025.

6. Cady, S.D.; Luo, W.; Hu, F.; Hong, M. Structure and function of the influenza a M2 proton channel. Biochemistry 2009, 48, 7356-7364.

7. Zoidis, G.; Kolocouris, N.; Kelly, J.M.; Prathalingam, S.R.; Naesens, L.; de Clercq, E. Design and synthesis of bioactive adamantanaminoalcohols and adamantanamines. Eur. J. Med. Chem. 2010, 45, 5022-5030.

8. Von Geldern, T.W.; Trevillyan, J.M. The next big thing in diabetes: Clinical progress on DPP-IV inhibitors. Drug Dev. Res. 2006, 67, 627-642.

9. Havale, S.H.; Pal, M. Medicinal chemistry approaches to the inhibition of dipeptidyl peptidase-4 for the treatment of type 2 diabetes. Bioorg. Med. Chem. 2009, 17, 1783-1802.

10. Zettl, H.; Schubert-Zsilavecz, M.; Steinhilber, D. Medicinal chemistry of incretin mimetics and DPP-4 inhibitors. ChemMedChem 2010, 5, 179-185.

11. Liu, J.; Obando, D.; Liao, V.; Lifa, T.; Codd, R. The many faces of the adamantyl group in drug design. Eur. J. Med. Chem. 2011, 46, 1949-1963.

12. Al-Omar, M.A.; Al-Abdullah, E.S.; Shehata, I.A.; Habib, E.E.; Ibrahim, T.M.; El-Emam, A.A. Synthesis, antimicrobial, and anti-inflammatory activities of novel 5-(1-adamantyl)-4-arylideneamino3-mercapto-1,2,4-triazoles and related derivatives. Molecules 2010, 15, 2526-2550.

13. Lamanna, G.; Russier, J.; Dumortier, H.; Bianco, A. Enhancement of anti-inflammatory drug activity by multivalent adamantane-based dendrons. Biomaterials 2012, 33, 5610-5617.

14. Antoniadou-Vyza, E.; Avramidis, N.; Kourounakis, A.; Hadjipetrou, L. Anti-inflammatory properties of new adamantane derivatives. Design, synthesis, and biological evaluation. Arch. Pharm. 1998, 331, $72-78$.

15. Priyanka, B.; Anitha, K.; Shirisha, S.; Dipankar, B.; Rajesh, K. Evaluation of anti-oxidant activity of ethanolic root extract of albizla lebbeck 1. Int. Res. J. Pharm. Appl. Sci. 2013, 3, 93-101.

16. Joyce, D.A. Oxygen radicals in disease. Advers. Drug React. Bull. 1987, 127, 476-479.

17. Farber, J.L. Mechanisms of cell injury by activated oxygen species. Environ. Health Perspect. 1994, 102, 17-24.

18. Lee, C.T.; Repasky, E.A. Opposing roles for heat and heat shock proteins in macrophage functions during inflammation: A function of cell activation state? Front. Immunol. 2012, 3, 1-7. 
19. Saso, L.; Valentini, G.; Casini, M.; Grippa, E.; Gatto, M.; Leone, M.; Silvestrini, B. Inhibition of heat-induced denaturation of albumin by nonsteroidal antiinflammatory drugs (NSAIDs): Pharmacological implications. Arch. Pharm. Res. 2001, 24, 150-158.

20. Kumar Chandraju Sadolalu, C.; Chia Tze, S.; Ooi Chin, W.; Quah Ching, K.; Chandraju, S.; Fun, H.K. Conformational studies of 2-(4-bromophenyl)-2-oxoethyl benzoates. Z. Kristallogr. Cryst. Mater. 2014, 229, doi:10.1515/zkri-2013-1709.

21. Jin, Y.; Guo, J.N.; Lin, K.; Tang, G.; Zhao, Y.F. Benzoylmethyl 4-chlorobenzoate. Acta Crystallogr. Sect. E Struct. Rep. Online 2008, 64, doi:10.1107/S1600536807066469/ng2408Isup2.hk1.

22. Komarov, I.V.; Gorichko, M.V.; Shishkin, O.V.; Kornilov, M.Y. Short communications-Unusual by-product in the bromination of 3,3-dibromocamphor. Russ. J. Org. Chem. 1999, 35, 1388-1389.

23. Fun, H.K.; Shahani, T.; Garudachari, B.; Isloor, A.M.; Satyanarayan, M.N. 2-(4-bromophenyl)-2oxoethyl 4-methylbenzoate. Acta Crystallogr. Sect. E Struct. Rep. Online 2011, 67, doi:10.1107/ S1600536811045272.

24. Fun, H.K.; Quah, C.K.; Vijesh, A.M.; Isloor, A.M.; Arulmoli, T. 2-(4-chlorophenyl)-2-oxoethyl 3,4-dimethoxybenzoate. Acta Crystallogr. Sect. E Struct. Rep. Online 2011, 67, doi:10.1107/ S1600536811048264.

25. Fun, H.K.; Chia, T.S.; Shenvi, S.; Isloor, A.M.; Garudachari, B. 2-(2,4-dichlorophenyl)-2-oxoethyl 4-methoxybenzoate. Acta Crystallogr. Sect. E Struct. Rep. Online 2011, 67, doi:10.1107/ S1600536811048720.

26. Fun, H.K.; Loh, W.S.; Garudachari, B.; Isloor, A.M.; Satyanarayan, M.N. 2-(4-fluorophenyl)-2oxoethyl 4-methoxybenzoate. Acta Crystallogr. Sect. E Struct. Rep. Online 2011, 67, doi:10.1107/ S1600536811049233.

27. Khan, I.; Ibrar, A.; Korzanski, A.; Kubicki, M. 2-(4-methylphenyl)-2-oxoethyl 3-bromobenzoate. Acta Crystallogr. Sect. E Struct. Rep. Online 2012, 68, doi:10.1107/S1600536812046995.

28. Chidan Kumar, C.S.; Yohannan Panicker, C.; Fun, H.-K.; Sheena Mary, Y.; Harikumar, B.; Chandraju, S.; Quah, C.K.; Ooi, C.W. Molecular structure, FT-IR, first order hyperpolarizability, NBO analysis, HOMO and LUMO analysis of 2-(4-chlorophenyl)-2-oxoethyl 3-methylbenzoate by $\mathrm{HF}$ and density functional methods. Spectrochim. Acta A 2014, 128, 327-336.

29. Chidan Kumar, C.S.; Panicker, C.Y.; Fun, H.-K.; Mary, Y.S.; Harikumar, B.; Chandraju, S.; Quah, C.K.; Ooi, C.W. FT-IR, molecular structure, first order hyperpolarizability, HOMO and LUMO analysis, MEP and NBO analysis of 2-(4-chlorophenyl)-2-oxoethyl 3-nitrobenzoate. Spectrochim. Acta A 2014, 126, 208-219.

30. Fun, H.K.; Arshad, S.; Garudachari, B.; Isloor, A.M.; Satyanarayan, M.N. 2-oxo-2-phenylethyl benzoate. Acta Crystallogr. Sect. E Struct. Rep. Online 2011, 67, doi:10.1107/S1600536811018976.

31. Fun, H.K.; Arshad, S.; Garudachari, B.; Isloor, A.M.; Satyanarayan, M.N. 2-(4-bromophenyl)-2oxoethyl 4-bromobenzoate. Acta Crystallogr. Sect. E Struct. Rep. Online 2011, 67, doi:10.1107/ S1600536811020654.

32. Fun, H.K.; Loh, W.S.; Garudachari, B.; Isloor, A.M.; Satyanarayan, M.N. 2-(4-bromophenyl)-2oxoethyl 4-methoxybenzoate. Acta Crystallogr. Sect. E Struct. Rep. Online 2011, 67, doi:10.1107/ S1600536811018988. 
33. Fun, H.K.; Loh, W.S.; Garudachari, B.; Isloor, A.M.; Satyanarayan, M.N. 2-(4-chlorophenyl)-2oxoethyl 3-(trifluoromethyl)benzoate. Acta Crystallogr. Sect. E Struct. Rep. Online 2011, 67, doi:10.1107/S1600536811020629.

34. Chidan Kumar, C.S.; Fun, H.K.; Tursun, M.; Ooi, C.W.; Chandraju, S.; Quah, C.K.; Parlak, C. Synthesis, molecular structure, FT-IR and XRD investigations of 2-(4-chlorophenyl)-2-oxoethyl 2-chlorobenzoate: A comparative DFT study. Spectrochim. Acta A 2014, 124, 595-602.

35. Fun, H.K.; Arshad, S.; Garudachari, B.; Isloor, A.M.; Satyanarayan, M.N. 2-(4-chlorophenyl)-2oxoethyl 2,4-difluorobenzoate. Acta Crystallogr. Sect. E Struct. Rep. Online 2011, 67, doi:10.1107/ S1600536811020630.

36. Fun, H.K.; Arshad, S.; Garudachari, B.; Isloor, A.M.; Shivananda, K.N. 2-(4-fluorophenyl)-2-oxoethyl 3-(trifluoromethyl)benzoate. Acta Crystallogr. Sect. E Struct. Rep. Online 2011, 67, doi:10.1107/ S160053681103947X.

37. Fun, H.K.; Asik, S.I.J.; Garudachari, B.; Isloor, A.M.; Satyanarayan, M.N. 2-(4-chlorophenyl)-2oxoethyl 2-methoxybenzoate. Acta Crystallogr. Sect. E Struct. Rep. Online 2011, 67, doi:10.1107/ S1600536811021246.

38. Fun, H.K.; Loh, W.S.; Garudachari, B.; Isloor, A.M.; Satyanarayana, M.N. 2-(4-bromophenyl)-2oxoethyl 4-hydroxybenzoate. Acta Crystallogr. Sect. E Struct. Rep. Online 2011, 67, doi:10.1107/ S1600536811040311.

39. Fun, H.K.; Yeap, C.S.; Garudachari, B.; Isloor, A.M.; Satyanarayan, M.N. 2-(4-bromophenyl)-2oxoethyl 4-chlorobenzoate. Acta Crystallogr. Sect. E Struct. Rep. Online 2011, 67, doi:10.1107/ S1600536811022963.

40. Fun, H.K.; Loh, W.S.; Garudachari, B.; Isloor, A.M.; Satyanarayana, M.N. 2-(4-chlorophenyl)-2oxoethyl 4-methylbenzoate. Acta Crystallogr. Sect. E Struct. Rep. Online 2011, 67, doi:10.1107/ S1600536811042851.

41. Fun, H.K.; Quah, C.K.; Garudachari, B.; Isloor, A.M.; Satyanarayan, M.N. 2-(4-bromophenyl)-2oxoethyl 2-methoxybenzoate. Acta Crystallogr. Sect. E Struct. Rep. Online 2011, 67, doi:10.1107/ S1600536811023002.

42. Fun, H.K.; Ooi, C.W.; Garudachari, B.; Isloor, A.M.; Satyanarayan, M.N. 2-(4-bromophenyl)-2oxoethyl 2-methylbenzoate. Acta Crystallogr. Sect. E Struct. Rep. Online 2011, 67, doi:10.1107/ S1600536811044564.

43. Fun, H.K.; Shahani, T.; Garudachari, B.; Isloor, A.M.; Shivananda, K.N. 2-(4-chlorophenyl)-2oxoethyl 4-hydroxybenzoate. Acta Crystallogr. Sect. E Struct. Rep. Online 2011, 67, doi:10.1107/ S1600536811037500.

44. Fun, H.K.; Shahani, T.; Garudachari, B.; Isloor, A.M.; Satyganarayan, M.N. 2-(4-chlorophenyl)2-oxoethyl benzoate. Acta Crystallogr. Sect. E: Struct. Rep. Online 2011, 67, doi:10.1107/ S160053681102383X.

45. Ji, T.; Wang, X.L.; Gao, Y.X.; Tang, G.; Zhao, Y.F. Benzoylmethyl 4-methoxybenzoate. Acta Crystallogr. Sect. E Struct. Rep. Online 2007, 63, doi:10.1107/S1600536807055250.

46. Isloor, A.M.; Garudachari, B.; Satyanarayan, M.N.; Gerber, T.; Hosten, E.; Betz, R. 2-(4-fluorophenyl)-2-oxoethyl 2-methoxybenzoate. Acta Crystallogr. Sect. E Struct. Rep. Online 2012, 68, doi:10.1107/S1600536812002577.

47. Bruker. Apex2, Saint and Sadabs; Bruker AXS Inc.: Madison, WI, USA, 2009. 
48. Sheldrick, G.M. A short history of shelx. Acta Cryst. 2008, 64, 112-122.

49. Dolomanov, O.V.; Bourhis, L.J.; Gildea, R.J.; Howard, J.A.; Puschmann, H. OLEX2: A complete structure solution, refinement and analysis program. J. Appl. Cryst. 2009, 42, 339-341.

50. Ruch, R.J.; Cheng, S.; Klaunig, J.E. Prevention of cytotoxicity and inhibition of intercellular communication by antioxidant catechins isolated from chinese green tea. Carcinogenesis 1989, 10, 1003-1008.

51. Farasat, M.; Khavari-Nejad, R.A.; Nabavi, S.M.B.; Namjooyan, F. Antioxidant activity, total phenolics and flavonoid contents of some edible green seaweeds from northern coasts of the persian gulf. Iran. J. Pharm. Res. 2014, 13, 163-170.

52. Ashok Kumar, B.S.; Saran, G.; Harshada, R.; Manoj, B.; Archana, P.G. Evaluation of anti-arthritic activity of vitex negundo by in vitro protein denaturation method. J. Tradit. Med. 2014, 1, 1-3.

Sample Availability: Samples of the compounds are available from the authors.

(C) 2015 by the authors; licensee MDPI, Basel, Switzerland. This article is an open access article distributed under the terms and conditions of the Creative Commons Attribution license (http://creativecommons.org/licenses/by/4.0/). 